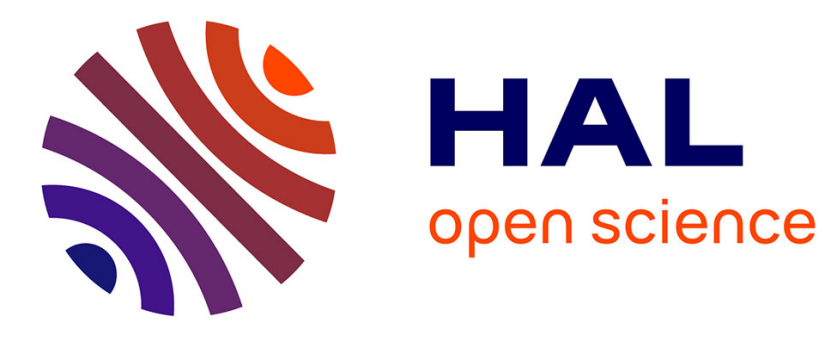

\title{
An interface-capturing method for incompressible two-phase flows. Validation and application to bubble dynamics
}

Thomas Bonometti, Jacques Magnaudet

\section{- To cite this version:}

Thomas Bonometti, Jacques Magnaudet. An interface-capturing method for incompressible two-phase flows. Validation and application to bubble dynamics. International Journal of Multiphase Flow, 2007, vol. 33, pp. 109-133. 10.1016/j.ijmultiphaseflow.2006.07.003 . hal-00862680

\section{HAL Id: hal-00862680 \\ https://hal.science/hal-00862680}

Submitted on 17 Sep 2013

HAL is a multi-disciplinary open access archive for the deposit and dissemination of scientific research documents, whether they are published or not. The documents may come from teaching and research institutions in France or abroad, or from public or private research centers.
L'archive ouverte pluridisciplinaire HAL, est destinée au dépôt et à la diffusion de documents scientifiques de niveau recherche, publiés ou non, émanant des établissements d'enseignement et de recherche français ou étrangers, des laboratoires publics ou privés. 


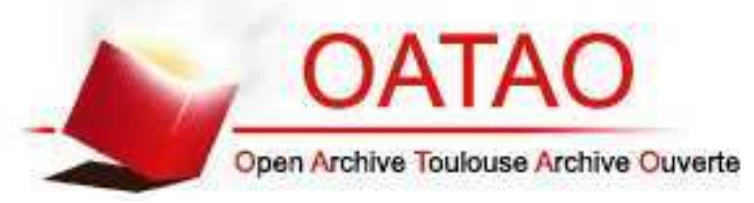

\section{Open Archive TOULOUSE Archive Ouverte (OATAO)}

OATAO is an open access repository that collects the work of Toulouse researchers and makes it freely available over the web where possible.

This is an author-deposited version published in : http://oatao.univ-toulouse.fr/ Eprints ID : 9406

To link to this article : DOI:10.1016/j.ijmultiphaseflow.2006.07.003 URL : http://dx.doi.org/10.1016/j.ijmultiphaseflow.2006.07.003

To cite this version : Bonometti, Thomas and Magnaudet, Jacques. An interface-capturing method for incompressible two-phase flows. Validation and application to bubble dynamics. (2007). International Journal of Multiphase Flow, vol. 33 (n ${ }^{\circ}$ 7). pp. 109-133. ISSN 0301-9322

Any correspondance concerning this service should be sent to the repository administrator: staff-oatao@ listes-diff.inp-toulouse.fr 


\title{
An interface-capturing method for incompressible two-phase flows. Validation and application to bubble dynamics
}

\author{
Thomas Bonometti *, Jacques Magnaudet \\ Institut de Mécanique des Fluides de Toulouse, UMR CNRS/INPT/UPS 5502, Allée Camille Soula, 31400 Toulouse, France
}

\begin{abstract}
We report on the development and applications of an interface-capturing method aimed at computing threedimensional incompressible two-phase flows involving high density and viscosity ratios, together with capillary effects. The numerical approach borrows some features to the Volume of Fluid method (since it is essentially based on the transport of the local volume fraction of the liquid) as well as to the Level Set technique (as no explicit reconstruction of the interface is carried out). The transport of the volume fraction is achieved by using a flux-limiting Zalesak scheme and the fronts are prevented from spreading in time by a specific strategy in which the velocity at nodes crossed by the interface is modified to keep the thickness of the transition region constant. As shown on several test cases, this algorithm allows the interface to deform properly while maintaining the numerical thickness of the transition region within three computational cells whatever the structure of the local flow field. The full set of governing equations is then used to investigate some fundamental aspects of bubble dynamics. More precisely we focus on the evolution of shape and rise velocity of a single bubble over a wide range of physical parameters and on head-on and side-by-side interactions between two rising bubbles.
\end{abstract}

Keywords: Bubbly flow; Front capturing; Interface tracking; Two-phase flow; Volume of Fluid

\section{Introduction}

The direct computation of incompressible two-phase flows has been an intense field of investigation over the last 15 years, as such flows are involved in many aspects of basic fluid mechanics as well as in engineering and environmental problems. One of the major technical issues in this area is to deal with changes in interface shape and topology. Numerical methods where interfaces freely evolve on a fixed grid have proved efficient for treating complex phenomena such as sloshing, splashing, breakup or coalescence. Several different techniques belonging to this wide family have been elaborated since the pioneering work of Harlow and Welch (1965). They may be divided within two main groups, depending on the way the interfaces are described.

\footnotetext{
* Corresponding author. Tel.: +33 5612859 07; fax: +33 561285991 .

E-mail addresses: thomas.bonometti@imft.fr (T. Bonometti), magnau@imft.fr (J. Magnaudet).
} 
One group, usually referred to as "Lagrangian techniques", makes use of markers to follow the interfaces. Front-Tracking methods (Unverdi and Tryggvason, 1992; De Sousa et al., 2004), and Marker methods (Popinet and Zaleski, 1999) belong to this class and introduce a secondary moving grid whose aim is to provide the interface location and curvature with a high accuracy. Particle-in-Cell methods (Harlow and Welch, 1965; Enright et al., 2002) that fill the domain near the interface with markers moving with the flow also belong to this group. These techniques become rather expensive as the number of markers increases. They may also encounter practical difficulties in managing the addition or deletion of markers as the interface is stretched or compressed by the flow (Unverdi and Tryggvason, 1992). The treatment of topological changes also requires special care. Recent developments concerning Front-Tracking methods (Juric and Tryggvason, 1998; Torres and Brackbill, 2000; Shin and Juric, 2002) improve this treatment and allow interfaces to merge or breakup quite naturally. However, these techniques remain quite difficult to implement for a general "blind" use.

The second group of methods is referred to as "Eulerian techniques". These techniques make use of a scalar function to define the location of the interface. This is especially the case of the Level Set method (Osher and Sethian, 1988; Sussman et al., 1994; Sethian, 1999) which is based on a smooth distance function whose zero value corresponds to the location of the interface. Level Set techniques classically suffer from poor mass conservation (Sussman et al., 1998). A way to improve this aspect is to locally modify the flow field within the interface region to keep the slope of the Level Set function constant, as suggested by Adalsteinsson and Sethian (1999). Other possibilities may consist in coupling the Level Set approach with a conservative method (Sussman and Puckett, 2000; Enright et al., 2002). Volume of Fluid methods (Hirt and Nichols, 1981; Youngs, 1982; Lafaurie et al., 1994) also belong to the group of Eulerian techniques. They transport the local volume fraction of one of the fluids, a property varying sharply from 0 to 1 across the interfacial region, to update the position of the interface. However, unlike the Level Set approach, they usually involve a step of effective interface reconstruction. This step is based on the local volume fraction and the orientation of its gradient. Interfaces are then generally reconstructed using a piecewise linear interpolation (Scardovelli and Zaleski, 1999). The main strength of the Volume of Fluid method is that it conserves mass exactly, as it works directly with the volume fraction. On the other hand, the main drawback of this method is the complexity of the reconstruction procedure in three dimensions.

The goal of the present paper is to describe some technical aspects and potentialities of a method which lies midway between the Volume of Fluid approach (as the transported quantity characterizing the two-phase nature of the flow is the volume fraction of one of the fluids) and the Level Set method (as no explicit reconstruction of the interface is involved). The development of this method was motivated by the well-known limitations of usual front-capturing techniques which, in the absence of an explicit interface reconstruction procedure, suffer unavoidably from spreading of fronts as the computation proceeds. Several numerical schemes aimed at overcoming this problem were proposed in the recent literature (Thuburn, 1996; Ubbink and Issa, 1999; Dendy et al., 2002). However, up to our knowledge, their capabilities have mostly been proven on simple transport tests rather than on real flow situations. Here we explore a simple alternative, described in Section 3, which consists in an improvement of existing techniques rather than in a radically new numerical scheme. This improved technique was developed to obtain a cheap and versatile tool for computing general three-dimensional two- and three-phase flows involving high density and viscosity contrasts, together with capillary effects. In Section 4 we quantify the performances of this technique on pure transport tests as well as on some basic situations governed by the full two-phase Navier-Stokes equations, such as bubble shape oscillations. Finally, we illustrate the potentialities of method in Section 5 by considering several subtle aspects of bubble dynamics.

\section{The Navier-Stokes equations and their numerical treatment}

We assume the two fluids to be Newtonian and incompressible, with uniform surface tension and no phase change. The evolution of the two-phase flow is then classically described using the one-fluid formulation of the Navier-Stokes equations, namely

$$
\frac{\partial \boldsymbol{V}}{\partial t}+\boldsymbol{V} \cdot \nabla \boldsymbol{V}=-\frac{1}{\rho} \nabla P+\boldsymbol{g}+\frac{1}{\rho} \nabla \cdot\left[\mu\left(\nabla \boldsymbol{V}+{ }^{t} \nabla \boldsymbol{V}\right)\right]-\frac{\sigma}{\rho}(\nabla \cdot \boldsymbol{n}) \boldsymbol{n} \delta_{\mathrm{I}} ; \quad \nabla \cdot \boldsymbol{V}=0
$$


where $\boldsymbol{V}, P, \rho$ and $\mu$ are the local velocity, pressure, density and dynamic viscosity in the flow, respectively, $g$ denotes gravity and $\sigma$ is the surface tension. The surface delta function $\delta_{\mathrm{I}}$ is zero outside the interface, the unit normal of which is denoted by $\boldsymbol{n}$. The local volume fraction of fluid 1 obeys

$$
\frac{\partial C}{\partial t}+\boldsymbol{V} \cdot \nabla C=0
$$

This volume fraction equals one (resp. zero) in cells filled with fluid 1 (resp. 2) and takes intermediate values in cells belonging to the transition region. The local density and dynamic viscosity are evaluated using a linear interpolation, namely

$$
\begin{aligned}
& \rho=C \rho_{1}+(1-C) \rho_{2} \\
& \mu=C \mu_{1}+(1-C) \mu_{2}
\end{aligned}
$$

Eq. (3a) is exact, as it may be obtained from a mass balance over a cell crossed by the interface. In contrast, Eq. ( $3 \mathrm{~b}$ ) is ad hoc and may lead to some physical inconsistencies, especially when an interface experiences a strong shear. A general model of the viscous stress tensor is derived and discussed in a forthcoming paper. Nevertheless (3b) will be used throughout the present study. The capillary force is transformed into a volume force using the Continuum Surface Force model proposed in Brackbill et al. (1992). Hence we write

$$
\frac{\sigma}{\rho}(\nabla \cdot \boldsymbol{n}) \boldsymbol{n} \delta_{\mathrm{I}}=\frac{\sigma}{\rho} \nabla \cdot\left(\frac{\nabla C}{\|\nabla C\|}\right) \nabla C
$$

The Navier-Stokes equations (1) and (2) are solved using the JADIM code developed in our group. Details on the spatial discretization and time-advancement algorithm used in this code for constant density situations may be found in several previous publications, especially Magnaudet et al. (1995), Calmet and Magnaudet (1997) and Legendre and Magnaudet (1998). Briefly, the momentum equations are discretized on a staggered orthogonal grid using a finite volume approach. The spatial discretization is performed using second-order centered differences. Time-advancement is achieved through a third-order Runge-Kutta algorithm for advective and source terms and a Crank-Nicolson algorithm for viscous stresses. Incompressibility is satisfied at the end of each time step through a projection method. The overall algorithm is second-order accurate in both time and space.

The main changes introduced in this procedure by the two-phase nature of the flows considered here are related to the variable density and viscosity and to the capillary force. Basically we start the time-advancement algorithm by solving (2), prior to solving the momentum equation. Hence, starting from $C^{n}(\boldsymbol{x})$ and $\boldsymbol{V}^{n}(\boldsymbol{x})$ at time $n \Delta t$, we first obtain the solution $C^{n+1}(\boldsymbol{x})$ corresponding to time $(n+1) \Delta t$ as discussed in Section 3 below, and use it to evaluate the density $\rho^{n+1}(\boldsymbol{x})$ and the viscosity $\mu^{n+1}(\boldsymbol{x})$ through (3). Then we define the secondorder approximations of $\rho$ and $\mu$ at time $(n+1 / 2) \Delta t$ as $\rho^{n+1 / 2}=\left(\rho^{n}+\rho^{n+1}\right) / 2, \mu^{n+1 / 2}=\left(\mu^{n}+\mu^{n+1}\right) / 2$ and use them throughout the time step $[n \Delta t,(n+1) \Delta t]$ to solve (1). Note that the volume fraction is defined at pressure nodes, so that linear interpolations are used to obtain the density and viscosity on velocity nodes. Finally the variable density projection method by which the final velocity field $\boldsymbol{u}^{n+1}$ is made divergence-free requires the solution of a pseudo-Poisson equation of the form $\nabla \cdot(\nabla \phi / \rho)=\nabla \cdot \boldsymbol{u}^{*}$ for the pressure increment $\phi, \boldsymbol{u}^{*}$ being the intermediate velocity field. The corresponding linear system is solved by a Jacobi preconditioned conjugate gradient technique. The JCG routine of the ITPACK Library is used for this purpose (a direct Cholesky method is also used in two-dimensional cases).

The capillary term (4) is evaluated by approximating $C$ at time $(n+1 / 2) \Delta t$ as $C^{n+1 / 2}=\left(C^{n}+C^{n+1}\right) / 2$. To evaluate the volume integral involved in the right-hand side of (4) over a computational cell bounded by a surface $\Gamma$ with a unit normal $\boldsymbol{N}$, we rewrite this term in the form $\sigma\left\{\int_{\Gamma} \boldsymbol{N} \cdot(\nabla C /\|\nabla C\|) \mathrm{d} \Gamma\right\} \overline{\nabla C / \rho}$, where $\overline{\nabla C / \rho}$ is a volume average of $\nabla C / \rho$ over the cell, all contributions being evaluated at velocity nodes using centered differences and second-order interpolations. As is well known, the sharp variations of $C$ throughout the transition region tend to generate spurious peaks in the curvature and lead to a poor evaluation of the capillary force. To avoid such artifacts, the capillary force is evaluated using a smoothed distribution $\widehat{C}$ of the volume fraction obtained from $C$ through several successive applications of a weighted average over the surrounding grid points. In two dimensions, the elementary smoothing procedure around a given grid point $(i, j)$ takes the form $\widehat{C}_{i, j}=3 / 4 C_{i, j}+\left(C_{i+1, j}+C_{i-1, j}+C_{i, j+1}+C_{i, j-1}\right) / 16$. Detailed tests concerning the influence of this smoothing 
procedure may be found in Bonometti (2005). Examples of the accuracy with which this method allows the shape of deforming bubbles to be predicted were given by Bonometti and Magnaudet (2006).

\section{Transporting the volume fraction}

\subsection{The general algorithm}

We basically solve Eq. (2) by using a modified version of the transport scheme proposed by Zalesak (1979). As is well known, this scheme belongs to the family of Flux Corrected Transport schemes (Boris and Book, 1973) initially derived for the treatment of shocks. Rudman (1997) and Benkenida (1999) among others noticed that the original multidimensional Zalesak scheme has a strong tendency to distort the fronts in several types of simple flows. To avoid this distortion, we split the transport equation (2) into three successive one-dimensional steps written in nonconservative form. For instance if the velocity components at time $n \Delta t$ are $U^{n}, V^{n}, W^{n}$ along directions $x, y$ and $z$, respectively, we successively advance the volume fraction from its value $C^{n}$ at time $n \Delta t$ to its value $C^{n+1}$ at time $(n+1) \Delta t$ through the explicit algorithm

$$
\begin{aligned}
& \frac{C^{*}-C^{n}}{\Delta t}+U^{n} \frac{\partial C^{n}}{\partial x}=0 \\
& \frac{C^{* *}-C^{*}}{\Delta t}+V^{n} \frac{\partial C^{*}}{\partial y}=0 \\
& \frac{C^{n+1}-C^{* *}}{\Delta t}+W^{n} \frac{\partial C^{* *}}{\partial z}=0
\end{aligned}
$$

where $C^{*}$ and $C^{* *}$ denote intermediate values of $C$. One-dimensional fluxes are evaluated in nonconservative form because the strain rates $\partial U / \partial x, \partial V / \partial y$ and $\partial W / \partial z$ may produce values of $C^{*}, C^{* *}$ or $C^{n+1}$ less than 0 or greater than 1 if the conservative form is used (Rudman, 1998). The diffusive/antidiffusive treatment of the spatial flux developed by Zalesak is applied during each of the above one-dimensional steps. This flux-limiting procedure which combines the use of a low-order and a high-order expression of the flux to guarantee the positivity and monotonicity of $C$ is second-order accurate. This algorithm is not detailed here, as it is extensively described in the original reference (Zalesak, 1979) as well as in several textbooks. Here we just mention that we use first- and eight-order approximations for the low- and high-order fluxes, respectively. The sequence along which the three spatial directions are treated is modified at every time step, so that no preferential direction is artificially created. Adding the three substeps (5a)-(5c) yields

$$
\frac{C^{n+1}-C^{n}}{\Delta t}+U^{n} \frac{\partial C^{n}}{\partial x}+V^{n} \frac{\partial C^{*}}{\partial y}+W^{n} \frac{\partial C^{* *}}{\partial z}=0
$$

Eq. (6) clearly indicates that, in contrast to the original multidimensional scheme in which $C^{*}=C^{* *}=C^{n}$, the splitting procedure yields an overall transport scheme which is not conservative anymore. Hence, a correction procedure has to be introduced to avoid small mass errors to accumulate and result in inaccurate long-time solutions. Controlling mass errors is a well-known issue encountered in both Lagrangian and Eulerian techniques, such as Boundary Elements (Zhou and Pozrikidis, 1993) and Front Tracking methods (Bunner and Tryggvason, 2002) whose transport algorithms are not explicitly conservative, but also in Level Set methods that use a redistanciation step (Sussman et al., 1998), or even in Volume of Fluid methods in which interface reconstruction is split into one-dimensional steps (Renardy et al., 2001).

The strategy adopted here for controlling mass errors is based on a modification of the local volume fraction $C$ that keeps the global mass of each fluid constant. A similar constraint was applied by Chang et al. (1996), Sussman and Uto (1998) and Spelt (2005) to improve the overall mass conservation of Level Set methods. Let $\Delta m(t)$ be the relative error of mass (or volume) in fluid 1 at time $t$ in the whole computational domain $\vartheta$. We have

$$
\Delta m(t)=\frac{\int_{\vartheta}(C(\boldsymbol{x}, t)-C(\boldsymbol{x}, t=0)) \mathrm{d} \vartheta}{\int_{\vartheta} C(\boldsymbol{x}, t=0) \mathrm{d} \vartheta}
$$




\section{a}

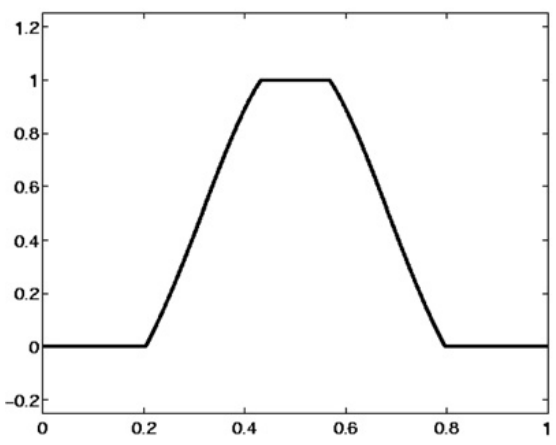

b

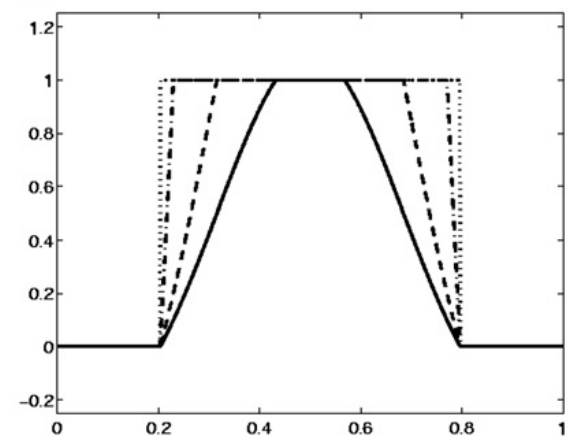

C

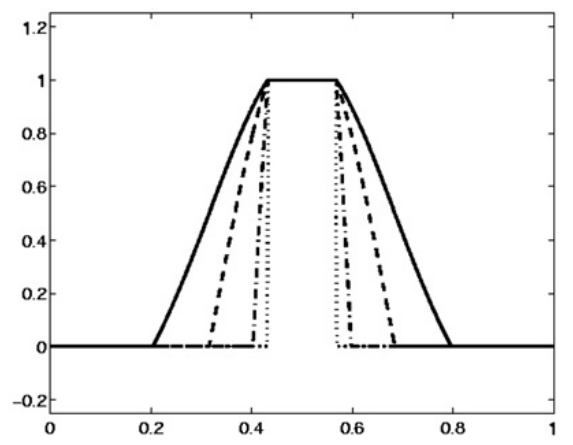

Fig. 1. Volume fraction modification to control mass errors. (a) $|\Delta m| \leqslant \Delta m_{\max }$; (b) $\Delta m<-\Delta m_{\max }$; (c) $\Delta m>\Delta m_{\max }$ ( (一) $\gamma=1$; (----) $\gamma=2 ;(-\cdot-\cdot-\cdot) \gamma=10 ;(\ldots \ldots) \gamma=10^{3}$.

The local volume fraction $C$ at any point $\boldsymbol{x}$ is replaced by a corrected volume fraction $\widetilde{C}(\boldsymbol{x}, t)$ defined as

$$
\widetilde{C}(\boldsymbol{x}, t)= \begin{cases}\min (\gamma C(\boldsymbol{x}, t), 1) & \text { if } \Delta m(t)<-\Delta m_{\max } \\ C(\boldsymbol{x}, t) & \text { if }|\Delta m(t)| \leqslant \Delta m_{\max } \\ 1-\min [\gamma(1-C(\boldsymbol{x}, t)), 1] & \text { if } \Delta m(t)>\Delta m_{\max }\end{cases}
$$

where $\gamma$ is a real parameter such that $\gamma \geqslant 1$. This parameter is computed iteratively until the criterion $|\Delta m(t)| \leqslant \Delta m_{\max }$ is satisfied. Based on extensive tests, we determined that the value $\Delta m_{\max }=10^{-3}$ is adequate, as in all cases we found the corresponding residual mass error to have no effect on the long-time dynamics of the flow. It is worth noting that Bunner and Tryggvason (2002) also found that a relative mass error of $10^{-3}$ did not induce any change in their results. The computational time required to evaluate $\widetilde{C}$ is less than $1 \%$ of the overall CPU time. In practice, as the overall mass error grows slowly, $C$ is modified only every 50 time steps approximately, and $\gamma$ converges in less than 10 iterations. Modifications of the volume fraction induced by the correction strategy (8) are illustrated in Fig. 1 where a one-dimensional distribution of $\widetilde{C}$ is shown for different values of $\gamma$. This figure makes it clear that the region where $0<\widetilde{C}<1$ is included within the transition region where the initial volume fraction $C$ is such that $0<C<1$. In addition (8) tends to stiffen the fronts, which is a positive feature. The most noticeable limitation of the above correction strategy is that it is global, since the mass error (7) is evaluated over the whole computational domain (as in Chang et al., 1996; Sussman and Uto, 1998; Spelt, 2005) rather than locally. Hence for instance in a dispersed flow, (8) may not strictly preserve the mass of each drop individually. Nevertheless, provided the logical tests required to identify the cells corresponding to each drop are performed, it is possible to define the equivalent of the relative mass error (7) for each of these subdomains. Therefore, with a moderate number of drops, the above method can be generalized so as to conserve the mass of the various subdomains of fluid 1, as in Bunner and Tryggvason (2002). Yet this correction strategy remains a first-order remedy, and further work needs to be done to obtain a technique capable of controlling mass errors in a local manner which deals explicitly with the specific geometry of the interface.

\subsection{Preserving the stiffness of the fronts}

In addition to the transport step, Volume of Fluid methods generally comprise an interface reconstruction step whose essential role is to prevent the fronts from spreading as time proceeds. As we did not wish to introduce such a reconstruction step in our method to keep it as cheap and flexible as possible, we were faced with this spreading problem. Level Set techniques do not escape the problem either, even though they define and maintain a zero-thickness interface through the zero isovalue of the Level Set function. This is because the Level Set function has to remain a signed distance throughout the computation and does not do so naturally, as it tends to spread in some regions of the flow and to bunch up in others. Different redistanciation techniques have been proposed to recover the required properties of the Level Set function (Sethian, 1999; Sussman et al., 1998; Adalsteinsson and Sethian, 1999). 
a

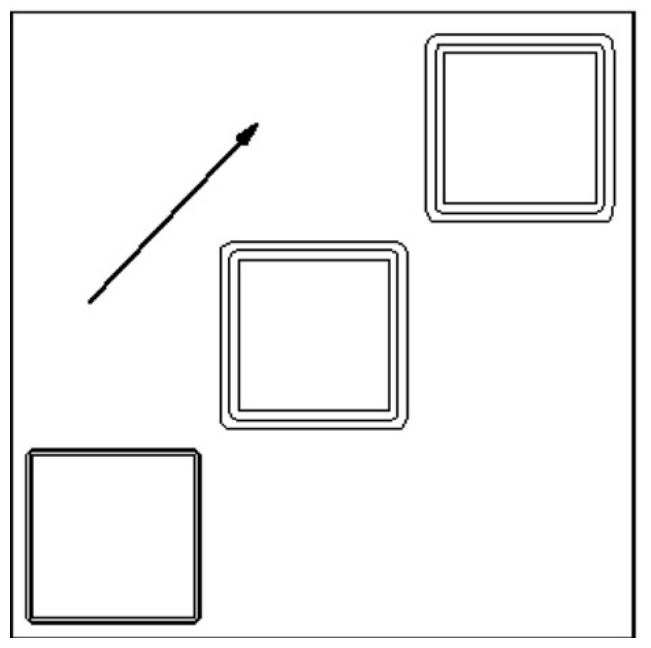

b

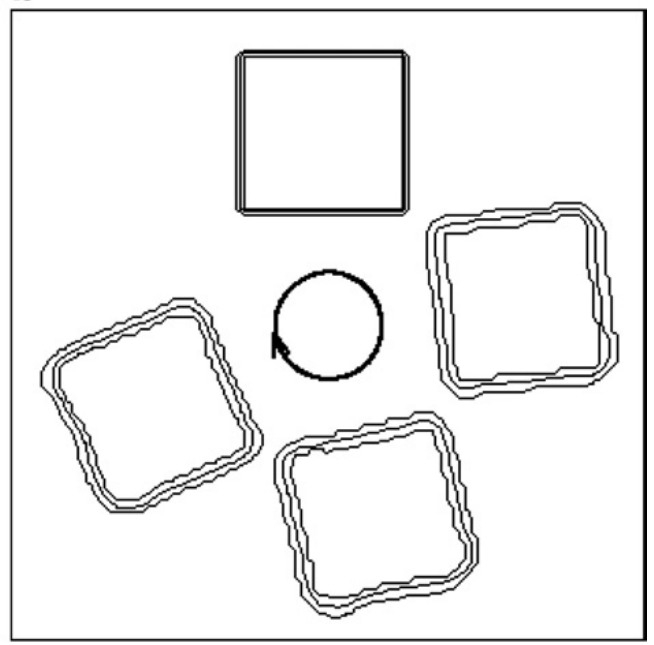

Fig. 2. Transport of the volume fraction using Zalesak scheme (iso- $C=0.01,0.5$ and $0.99,100 \times 100$ grid). (a) Translation $U=V=1$; (b) rotation $U=-y, V=x$.

The transport scheme described in Section 3.1 preserves the stiffness of the fronts in presence of a translation, whatever its orientation relative to the grid, as shown in Fig. 2. Clearly the front thickness displayed in Fig. 2(a) (defined as the distance separating the iso- $C$ lines corresponding to $C=0.99$ and $C=0.01$, respec-

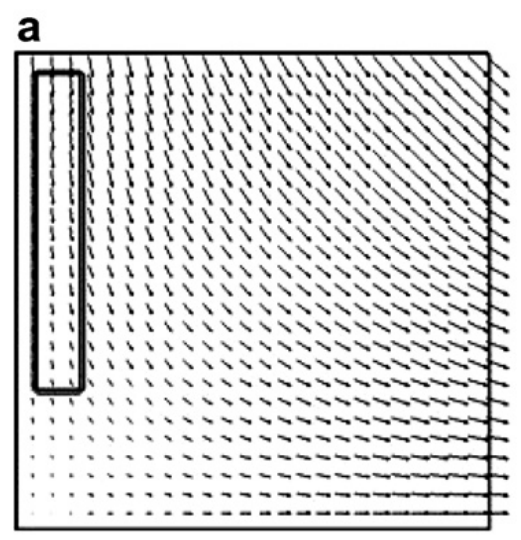

b
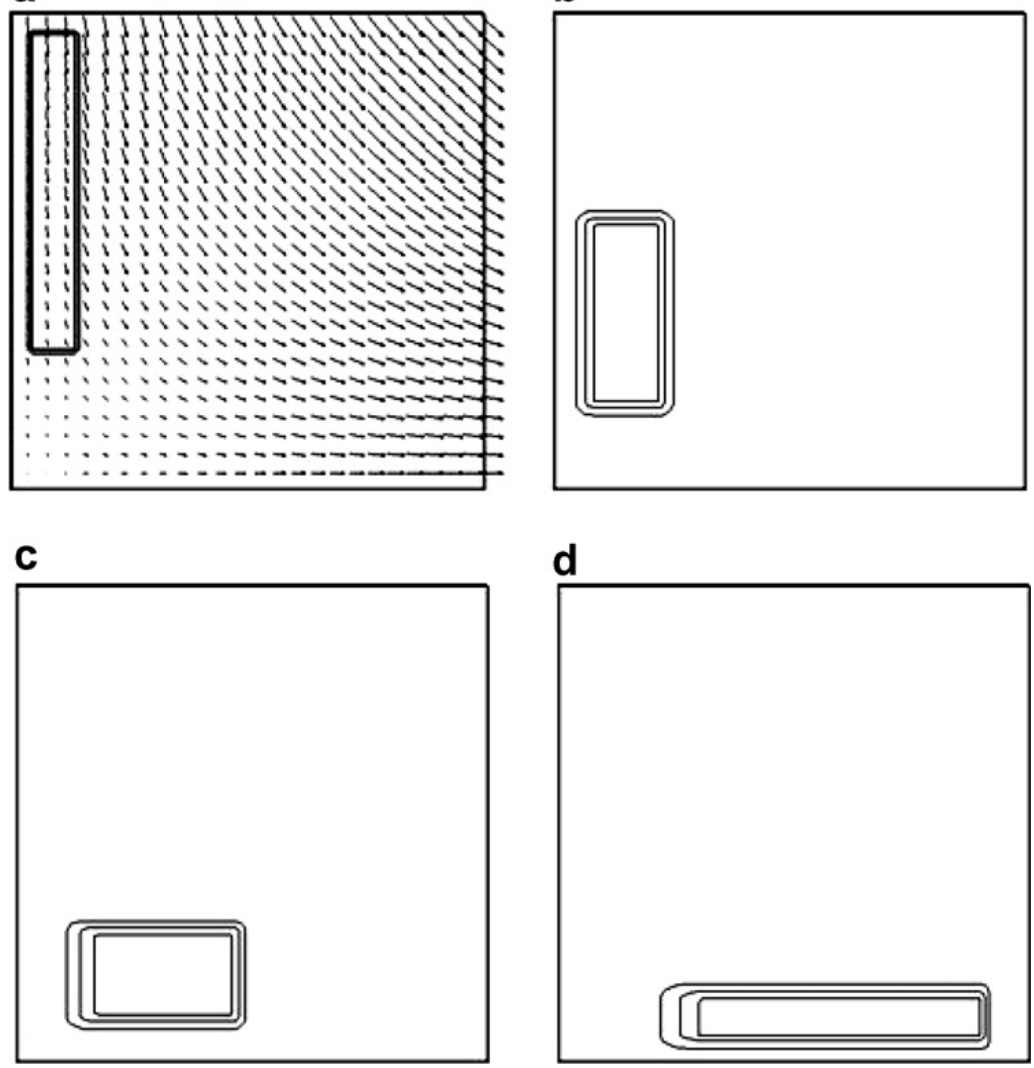

d

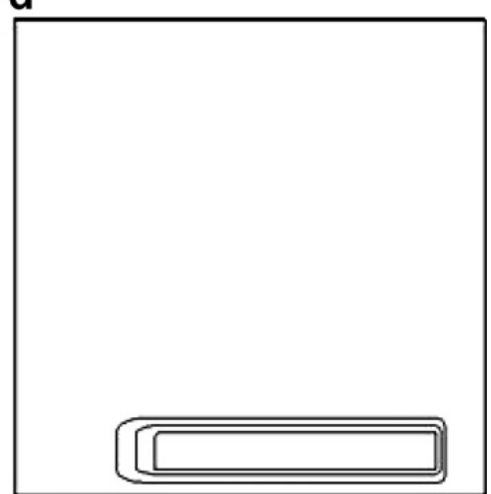

Fig. 3. Transport of the volume fraction in a pure straining flow $U=a x, V=-a y$ (here $a=1$ ), using Zalesak scheme (iso- $C=0.01,0.5$ and $0.99,100 \times 100$ grid). 
tively) is limited to three grid cells at all time. The same conclusion holds in presence of a solid body rotation, as shown in Fig. 2(b) (note however that the corners suffer from some erosion).

In contrast, Fig. 3 is very revealing of situations in which the front may spread dramatically. The components of the corresponding velocity field $V$ are $U=a x, V=-a y(a>0)$, with $x=y=0$ at the lower left corner. The eigenvalues of $\nabla \boldsymbol{V}$ are thus $a$ and $-a$ and the corresponding eigenvectors $\lambda_{a}$ and $\lambda_{-a}$ are the unit vectors $e_{1}$ and $e_{2}$ parallel to the grid lines $y=$ const. and $x=$ const., respectively. The rectangle first undergoes a compression along the $y$-axis (Fig. 3(a)) and transforms into a square when it comes close to the hyperbolic point $x=y=0$ (Fig. 3(b)). This stage mostly maintains the stiffness of the fronts. Then the square moves apart from the hyperbolic point and starts to undergo an elongation along the $x$-axis (Fig. 3(c)), eventually returning to its initial shape with the horizontal and vertical axes interchanged (Fig. 3(d)). While the final thickness of the fronts is still reasonable on the upper, lower and right sides of the rectangle, it has increased dramatically on the left side. This situation tells us several things. First, we note that the fronts corresponding to the horizontal sides of the rectangle always remain stiff. These fronts are all orthogonal to $\lambda_{-a}$ (i.e. they are compressed), but they are either perpendicular to the local velocity $\boldsymbol{V}$ (as in Fig. 3(a)) or parallel to it (as in Fig. 3(d)). This allows us to conclude that compressed fronts remain stiff, irrespective of the angle they make with the fluid velocity. Second, we see that in Fig. 3(a) and (b) the fronts corresponding to the vertical sides of the rectangle do not spread, whereas in Fig. 3(c) and (d), the left side of the rectangle does. These fronts are all orthogonal to $\lambda_{a}$. However in the first two cases the local velocity $\boldsymbol{V}$ is mostly parallel to the front, whereas it is mostly orthogonal to it in the last two ones. Hence it turns out that the fronts spread when they are orthogonal to both $\boldsymbol{V}$ and $\boldsymbol{\lambda}_{a}$. Finally, it is obvious from Fig. 3(d) that the spreading is much larger on the left side than on the right one, which emphasizes the importance of the characteristic stretching length $\delta=\left(U^{2}+V^{2}\right)^{1 / 2} / a$; the smaller $\delta$, the larger the spreading. To summarize, Fig. 3 tells us that the most dangerous situation regarding the spreading of fronts is encountered in regions where the front is crossed by a weak flow with a large elongation rate.

Our strategy to deal with the spreading problem borrows some aspects from recent improvements of Level Set methods (Adalsteinsson and Sethian, 1999; Gomes and Faugeras, 2000) in which the authors tackled the issue of maintaining the gradient of the Level Set function unity by modifying locally the velocity field near the interface. Here, we basically seek to remove the stretching within the transition region by making the velocity field locally constant across it, as illustrated in Fig. 4. To this end, we consider successively each of the one-dimensional steps of (5) during which $C$ is advected along the $i$ th direction of the grid $(i=1,3)$. Let then $\Omega_{i}$ be the transition region corresponding to points such that $0<C<1$ at the beginning of this step. Our method consists in advecting the front by a locally modified velocity field $\widetilde{\boldsymbol{V}}$ evaluated from $\boldsymbol{V}$ as follows:

$$
\widetilde{V}_{i}(\boldsymbol{x}, t)= \begin{cases}V_{i}(\boldsymbol{x}, t) & \text { if } x \notin \Omega_{i} \\ V_{i}\left(\boldsymbol{x}_{0}, t\right) & \text { if } x \in \Omega_{i}\end{cases}
$$

where $x_{0}$ lies on the iso- $C$ line $C=0.5$ and is the point closest to $\boldsymbol{x}$ that lies either on the $i$ th grid line or on the streamline passing through $\boldsymbol{x}$, depending on which of them makes the smallest angle with the local

a

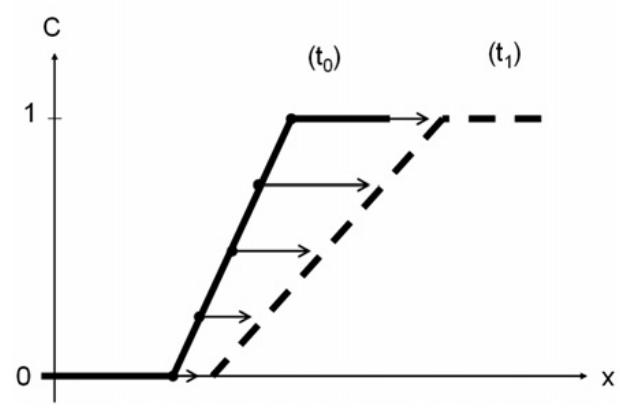

b

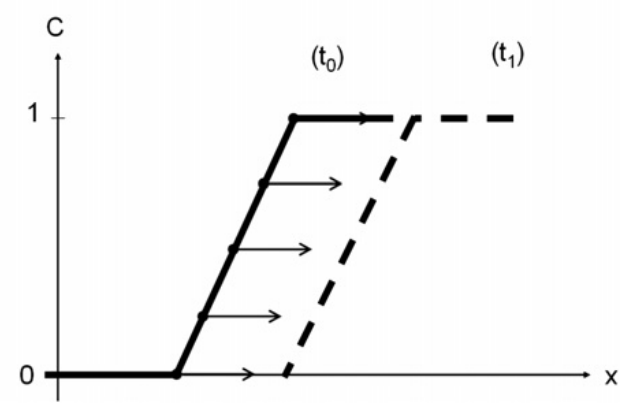

Fig. 4. Schematic of the strategy followed to preserve the stiffness of the fronts. (a) Transport with the flow velocity $V$ and (b) transport with a locally modified velocity $\widetilde{\boldsymbol{V}}$. Note that $\widetilde{\boldsymbol{V}}$ equals $\boldsymbol{V}(C=0.5)$. 
volume fraction gradient. As mentioned above, this procedure is repeated independently during each of the one-dimensional steps of (5). Obviously the mass of each fluid is not conserved during the application of (9). However the corresponding error may be cumulated with that resulting from the one-dimensional splitting of the transport algorithm and the whole mass error is treated through the procedure described in Section 3.1. We found that the computational time spent to evaluate $\widetilde{\boldsymbol{V}}$ using (9) is less than $2 \%$ of the total CPU time in all the computations we performed so far. Obviously the modified velocity field $\widetilde{\boldsymbol{V}}$ is only used in the computation of $C$, while the real velocity field $\boldsymbol{V}$ remains the only one involved in the Navier-Stokes equations (1).

Let us now detail the above choices concerning the way $\boldsymbol{x}_{0}$ is selected.

\subsubsection{The threshold condition $C\left(\boldsymbol{x}_{0}\right)=0.5$}

Since the thickness of the transition region may not be less than three computational cells, the velocity is in general nonuniform across it and evolves smoothly from one value on the side of $\Omega_{i}$ corresponding to $C \rightarrow 1$, to another value on the side corresponding to $C \rightarrow 0$. The true value of the interface velocity, which does not depend on the discretization, lies between these two extreme values. Fig. 5 shows how the algorithm described in Section 3.1 transports a step of $C$ in a stretching flow on different grids. It is clear from this figure that the location of the value $C=0.5$ is independent of the grid and coincides with the theoretical location of the jump, while the location of all other values of $C$ is not. This allows us to conclude that the physical value of the interface velocity is that corresponding to $C=0.5$ and leads us to select $\boldsymbol{x}_{0}$ such that $C\left(\boldsymbol{x}_{0}\right)=0.5$.

\subsubsection{Selecting the direction of $\boldsymbol{x}-\boldsymbol{x}_{0}$}

For any point $\boldsymbol{x}$ of the transition region $\Omega_{i}$, the direction of $\boldsymbol{x}-\boldsymbol{x}_{0}$ may be chosen among several candidates. As the above discussion emphasizes the role of the orientation of the front (defined by $\nabla C$ ) with respect to the local velocity $\boldsymbol{V}$ and to the eigenvectors of $\nabla \boldsymbol{V}$, it seems natural to use the direction provided by one of these quantities to define $\boldsymbol{x}-\boldsymbol{x}_{0}$. However the eigenvectors of $\nabla \boldsymbol{V}$ have an imaginary part when the flow is locally elliptic rather than hyperbolic, so that they cannot be used easily in a general method which has to deal with all types of flows. On the other hand, the iso-coordinates $x_{i}=$ const. of the grid also play a particular role in the one-dimensional splitting (5), as they are used to advance the volume fraction in time. Consequently, as shown on a particular example in Fig. 6, we are left with three simple possibilities.

The most obvious choice is to align $\boldsymbol{x}-\boldsymbol{x}_{0}$ with the direction of the volume fraction gradient. However, errors in the evaluation of $\nabla C$ mostly occur in regions of high curvature and may lead to errors in the determination of $\boldsymbol{x}_{0}$. Several tests revealed that this choice does not allow the shape of highly curved regions to be properly maintained, which led us to conclude that selecting the $\nabla C$-direction is not appropriate (see Fig. 10(c) for an illustration of how this transport strategy behaves in a real situation). Forcing $\boldsymbol{x}_{-} \boldsymbol{x}_{0}$ to

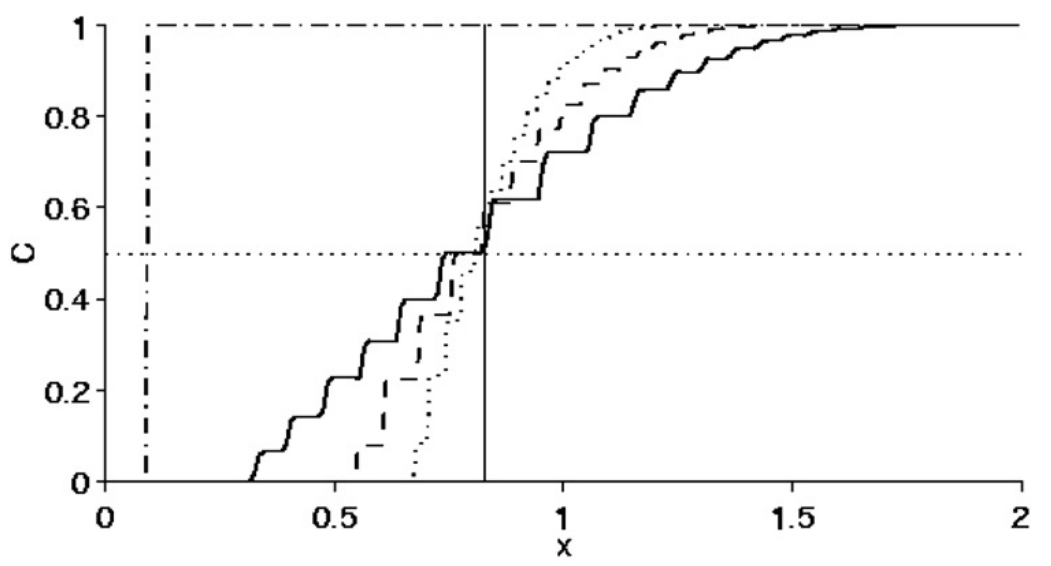

Fig. 5. Transport of a step of $C$ by the velocity field $U(x)=\exp \left[-5 \exp \left(-25 x^{2}\right)\right]$. (------) Initial condition; (-) theoretical solution at time $t=3.5$. Results obtained on different grids with the initial scheme $(\widetilde{\boldsymbol{V}}=\boldsymbol{V})$ at time $t=3.5$ : $(-)$ coarse grid $(\Delta x=1 / 100) ;(----)$ medium grid $(\Delta x=1 / 200) ;(\ldots \ldots)$ fine grid $(\Delta x=1 / 400)$. 

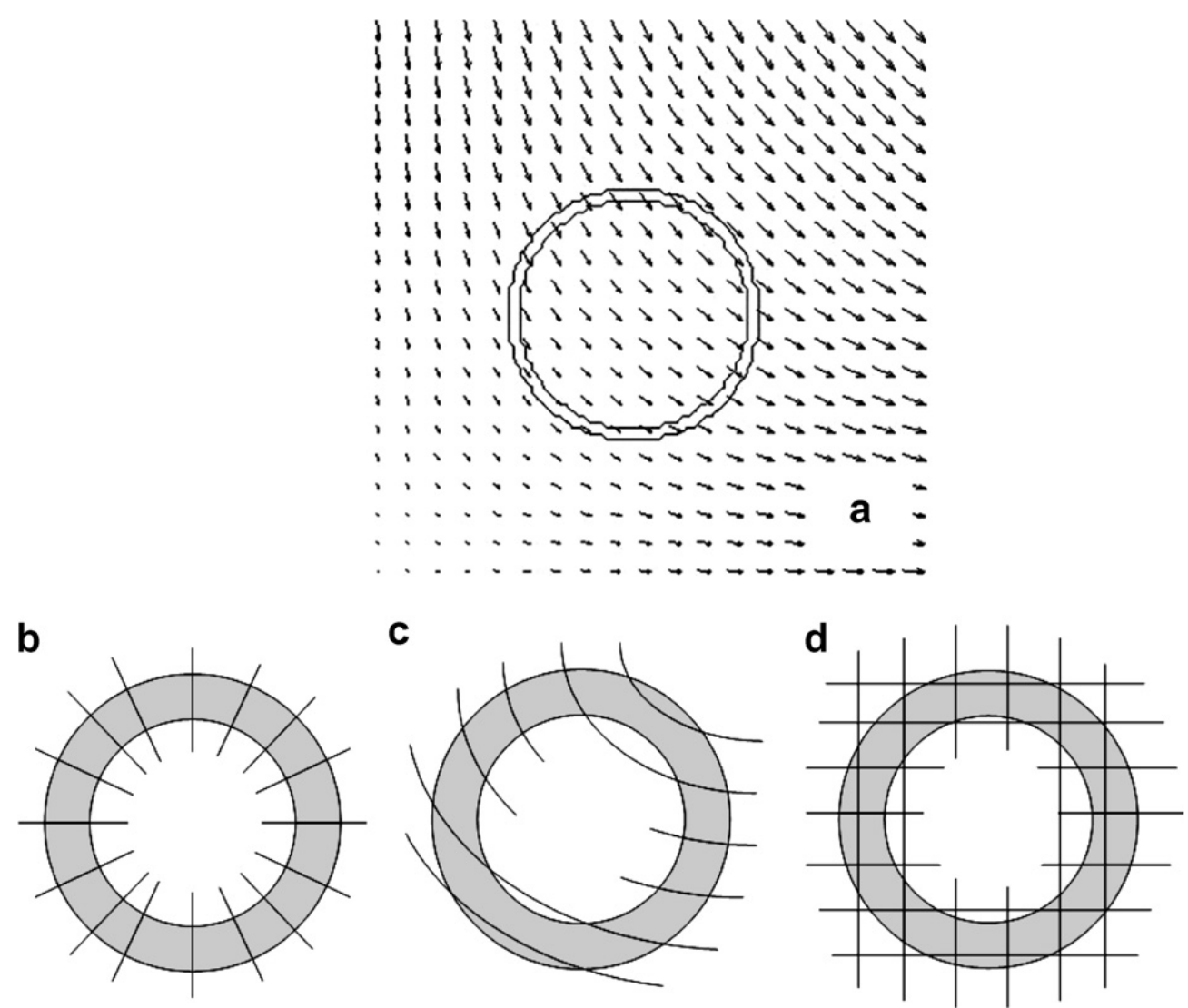

Fig. 6. The candidate directions for the determination of $\boldsymbol{x}_{0}$. (a) A circular interface in a stretching flow. (b) directions parallel to $\nabla C$; (c) streamlines; (d) grid lines. The grey zone represents the transition region $\Omega$.

be parallel everywhere to the direction of the local streamline is another option which provides good results in regions where the flow crosses the front. However it cannot be used alone since the problem of finding $C\left(\boldsymbol{x}_{0}\right)=0.5$ such that $\boldsymbol{x}-\boldsymbol{x}_{0}$ is parallel to $\boldsymbol{V}(\boldsymbol{x})$ has no solution in regions where the front is parallel to $\boldsymbol{V}$. Moreover, near such regions, the solutions $\boldsymbol{x}_{0}$ and $\boldsymbol{x}_{0}^{\prime}$ corresponding to two neighboring points $\boldsymbol{x}$ and $\boldsymbol{x}^{\prime}$ may be widely separated, leading to large differences between $\widetilde{\boldsymbol{V}}\left(\boldsymbol{x}_{0}\right)$ and $\widetilde{\boldsymbol{V}}\left(\boldsymbol{x}_{0}^{\prime}\right)$. Rather than improving the global result, this undesirable behavior may dramatically worsen it in some cases. Another possibility consists in choosing $\boldsymbol{x}_{0}$ such that $\boldsymbol{x}-\boldsymbol{x}_{0}$ is parallel to the $i$ th grid direction along which the volume fraction is advected during the current one-dimensional step of (5). This choice is obviously consistent with the transport algorithm. Based on extensive tests, it turned out to be the most efficient in most situations, as it offers two or three (depending on whether the computation is $2 \mathrm{D}$ or $3 \mathrm{D}$ ) different directions to find $\boldsymbol{x}_{0}$. However in regions where neither the front, nor the velocity is locally parallel to one of the grid lines, this strategy may become inefficient (see e.g. Fig. 10(b)).

The above considerations led us to adopt a mixed strategy which keeps the best of the above two possibilities. For this purpose, at any point $\boldsymbol{x}$ of $\Omega_{i}$, we evaluate the dot product $\alpha_{i}=(\nabla C /\|\nabla C\|) \cdot \boldsymbol{e}_{i}$, where $\boldsymbol{e}_{i}$ is the unit vector corresponding to the $i$ th direction. Similarly we evaluate the dot product $\beta_{i}=(\nabla C /\|\nabla C\|)$. $(\boldsymbol{V} /\|\boldsymbol{V}\|)$, where $\boldsymbol{V}$ is the local velocity. Then, depending on which of $\left|\alpha_{i}\right|$ and $\left|\beta_{i}\right|$ is the smallest, we seek $\boldsymbol{x}_{0}$ either on the grid line issuing from $\boldsymbol{x}$ and parallel to $\boldsymbol{e}_{i}$ or on the streamline issuing from $\boldsymbol{x}$. Note that in certain particular situations, especially when the streamline and the $i$ th grid line are both parallel to the front, it may happen that during a given one-dimensional step none of the above two families of lines crosses the curve $C=0.5$. In such cases we simply maintain $\widetilde{\boldsymbol{V}}_{i}=\boldsymbol{V}_{i}$.

In practice the procedure used to find $\boldsymbol{x}_{0}$ comprises several steps. It first consists in evaluating $\alpha_{i}(\boldsymbol{x})$ and $\beta_{i}(\boldsymbol{x})$ and checking whether $C(\boldsymbol{x})$ is smaller or larger than 0.5 . Then we examine the values of $C$ at the two grid points which, among the closest neighbors of $\boldsymbol{x}$, are located either on the same $i$ th grid line (if $\left|\alpha_{i}\right| \geqslant\left|\beta_{i}\right|$ ) 

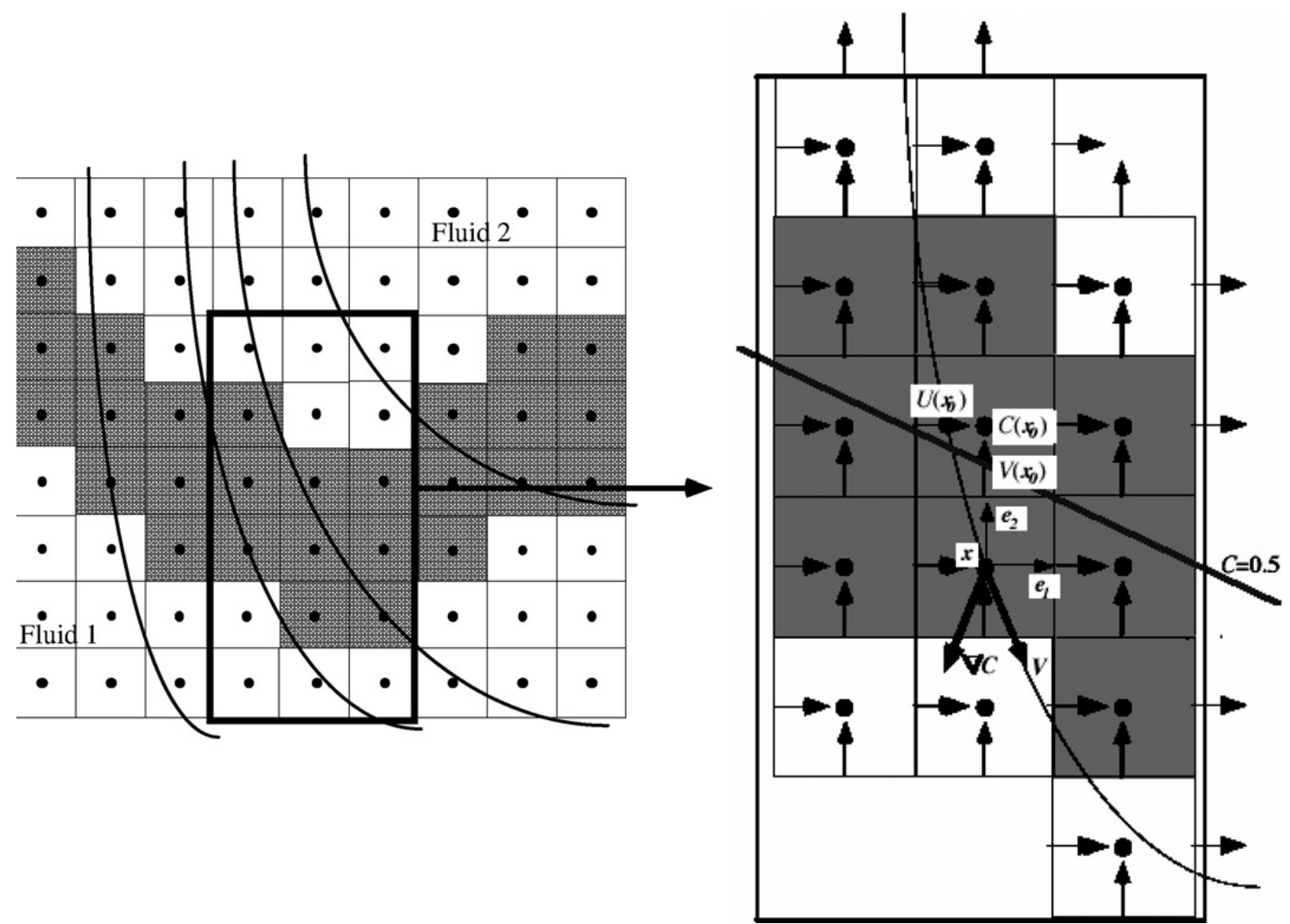

Fig. 7. An example of the selection process of $\widetilde{\boldsymbol{V}}$. The grey area represents the transition region $\Omega_{i}$, the curves symbolize the streamlines. At point $\boldsymbol{x}$ we have $\left|\alpha_{1}\right|<|\beta|$ and $\left|\alpha_{2}\right|>|\beta|$. Consequently, if $i=1, \boldsymbol{x}_{0}$ is seeked along the streamline, resulting in $\widetilde{U}(\boldsymbol{x})=U\left(\boldsymbol{x}_{0}\right)$. In contrast, if $i=2, x_{0}$ is seeked along the grid line $x=$ const. resulting in $\widetilde{V}(\boldsymbol{x})=V\left(x_{0}\right)$.

or are the closest to the straight line parallel to $\boldsymbol{V}(\boldsymbol{x})$ issuing from $\boldsymbol{x}$ (if $\left|\beta_{i}\right|>\left|\alpha_{i}\right|$ ). Let us suppose that we found $C(\boldsymbol{x})<0.5$. Then, if none of the above two values of $C$ is larger than $C(\boldsymbol{x})$, we simply keep $\boldsymbol{V}_{i}(\boldsymbol{x})=\boldsymbol{V}_{i}(\boldsymbol{x})$. If at least one of them exceeds $C(x)$, we pin the corresponding point $x_{1}$ (or the point corresponding to the largest of the two values if they are both larger than $C(\boldsymbol{x}))$ and restart the procedure with $\boldsymbol{x}_{1}$ in place of $\boldsymbol{x}$. The procedure is repeated $n$ times until we find $C\left(\boldsymbol{x}_{n}\right)>0.5$. Using a linear interpolation between $\boldsymbol{x}_{n-1}$ and $\boldsymbol{x}_{n}$ we then determine the approximate location $x_{0}$ such that $C\left(x_{0}\right)=0.5$ and by another linear interpolation evaluate $\widetilde{\boldsymbol{V}}_{i}(\boldsymbol{x})=\boldsymbol{V}_{i}\left(\boldsymbol{x}_{0}\right)$. The procedure follows symmetrically in the case $C(\boldsymbol{x})>0.5$. An illustration of the way the above algorithm works is provided in Fig. 7.

\subsection{Summary}

To sum up, the transport algorithm comprises the following steps:

- along each spatial direction (the order in which they are treated is interchanged at every time step):

(a) detect the transition region $\Omega_{i}$ and remove the strain rate within it by replacing $V_{i}$ by $\widetilde{\boldsymbol{V}}_{i}$ as described above,

(b) advance $C$ by solving the one-dimensional transport equation (5) along the $i$ th direction using $\widetilde{\boldsymbol{V}}_{i}$. Fluxes $\widetilde{\boldsymbol{V}}_{i} \partial C / \partial x_{i}$ (no summation on $i$ ) are evaluated using Zalesak scheme,

- control the computed mass errors by calculating the modified volume fraction $\widetilde{C}$ through (8).

In spite of its apparent complexity, the above technique is simple to implement and works in a fully automatic manner. Moreover the extension from 2D to 3D flows is straightforward, in contrast to the interface 
reconstruction step used in classical VOF approaches. Finally, as already indicated, the corresponding computational cost is marginal.

\section{Numerical tests}

\subsection{Tests of the transport algorithm}

We first present three tests of the whole transport procedure described in Section 3 in several nonuniform flows. We do not reconsider the cases of pure translation and solid body rotation, as the correction procedure leaves the corresponding results essentially unchanged compared to those shown in Fig. 2. Only Eqs. (5) are solved here, using a prescribed velocity field. Results obtained with the correction strategy described in Section 3.2 are compared with those provided by the original scheme $(\widetilde{\boldsymbol{V}}=\boldsymbol{V})$. We only show one- and two-dimensional test cases but three-dimensional cases were run as well and yielded similar results.

We first come back to the one-dimensional straining flow used in Fig. 5. A one-dimensional step of $C$ is transported by a straining flow $U(x)=\exp \left[-5 \exp \left(-25 x^{2}\right)\right]$ that creates a region of high strain followed by a nearly uniform region (Fig. 8(a) and (b)). Fig. 8(c) and (d) shows how $C(x, t)$ evolves in time with the initial transport scheme (Fig. 8(c)) and the modified one (Fig. 8(d)). In the first case the front spreads over many computational cells and this spreading increases in time. In contrast the modified scheme allows the front to remain sharp at all time. In Fig. 8(d) it may be noticed that the front first spreads over three grid cells in the stretching region (see the diamond symbols), and then contracts in the nearly-uniform region. The differences between the computed and theoretical positions of the front are less than one $\Delta x$. Then we reconsider the example of Fig. 3 which provided the basis of the correction strategy developed in Section 3.2. Unlike in Fig. 9(c) where the vertical fronts spread over roughly 10 cells, the solution obtained with the modified scheme (Fig. 9(d)) is found to preserve the stiffness of all fronts, allowing the position and shape of the final rectangle to agree well with the theoretical solution of Fig. 9(b).
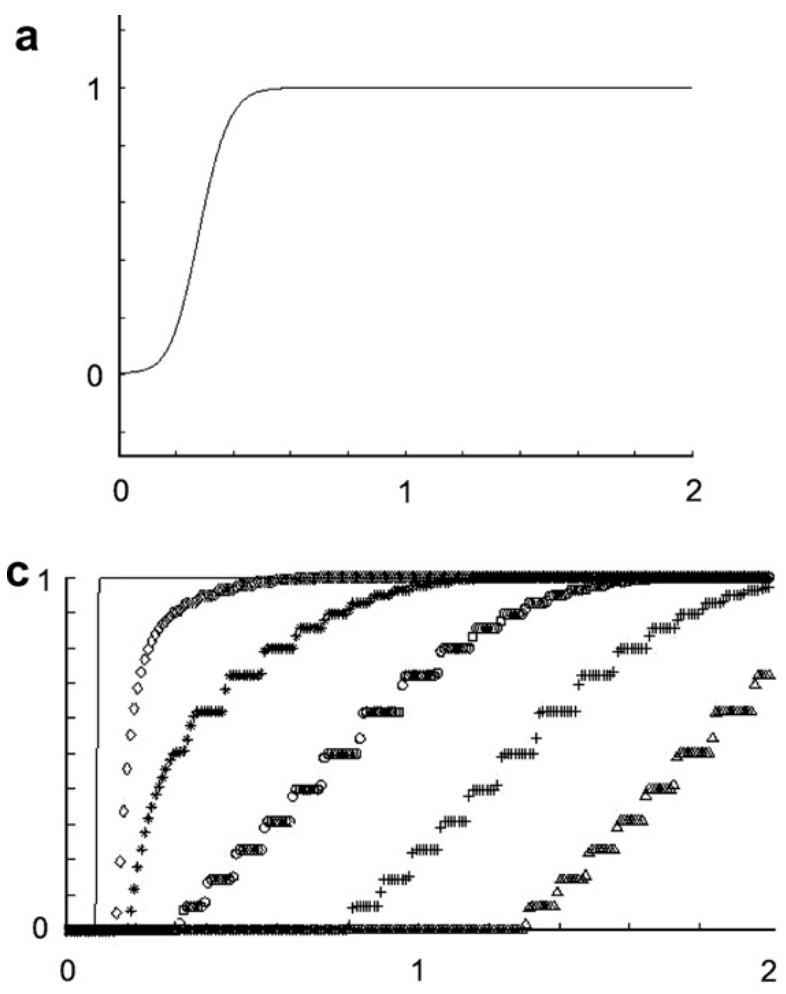

b
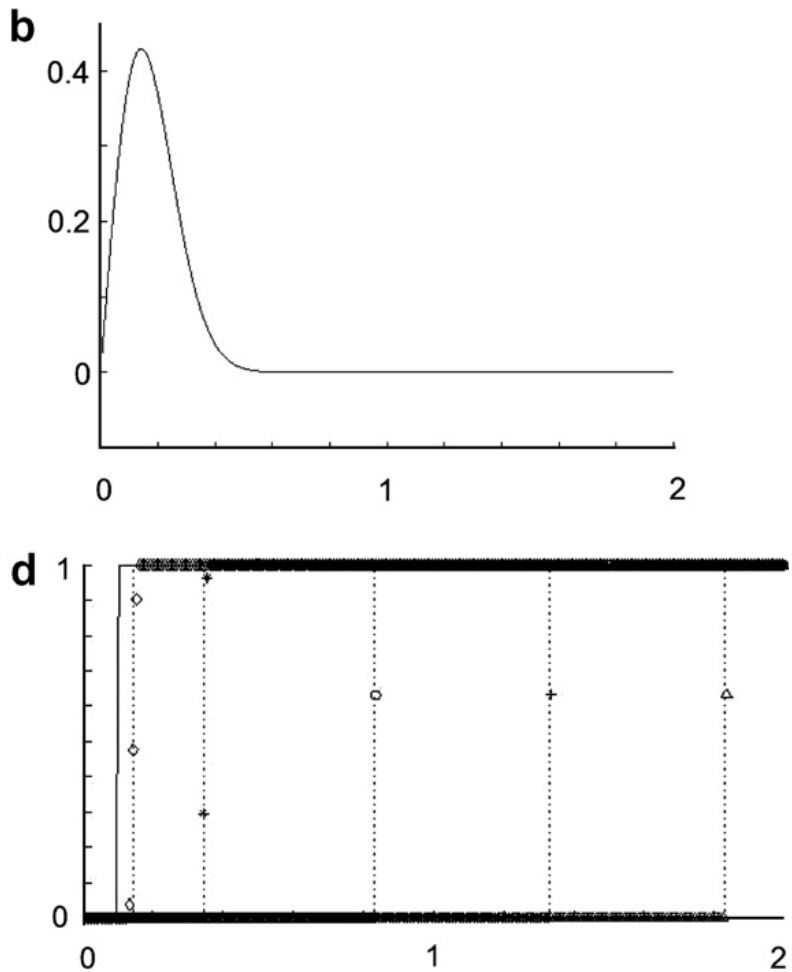

Fig. 8. Advection of a step of $C$ by a one-dimensional straining flow. (a) Velocity $U(x)$; (b) dimensionless strain rate $S_{x}(x)=(\Delta x / U) \mathrm{d} U /$ $\mathrm{d} x$; (c) evolution of $\mathrm{C}(x, t)$ obtained using $\boldsymbol{V}$. (d) Evolution of $\mathrm{C}(x, t)$ obtained using $\widetilde{\boldsymbol{V}}$. (一) Initial position; (.....) theoretical solution. The symbols indicate the numerical solution $(\Delta x=1 / 100)$. 

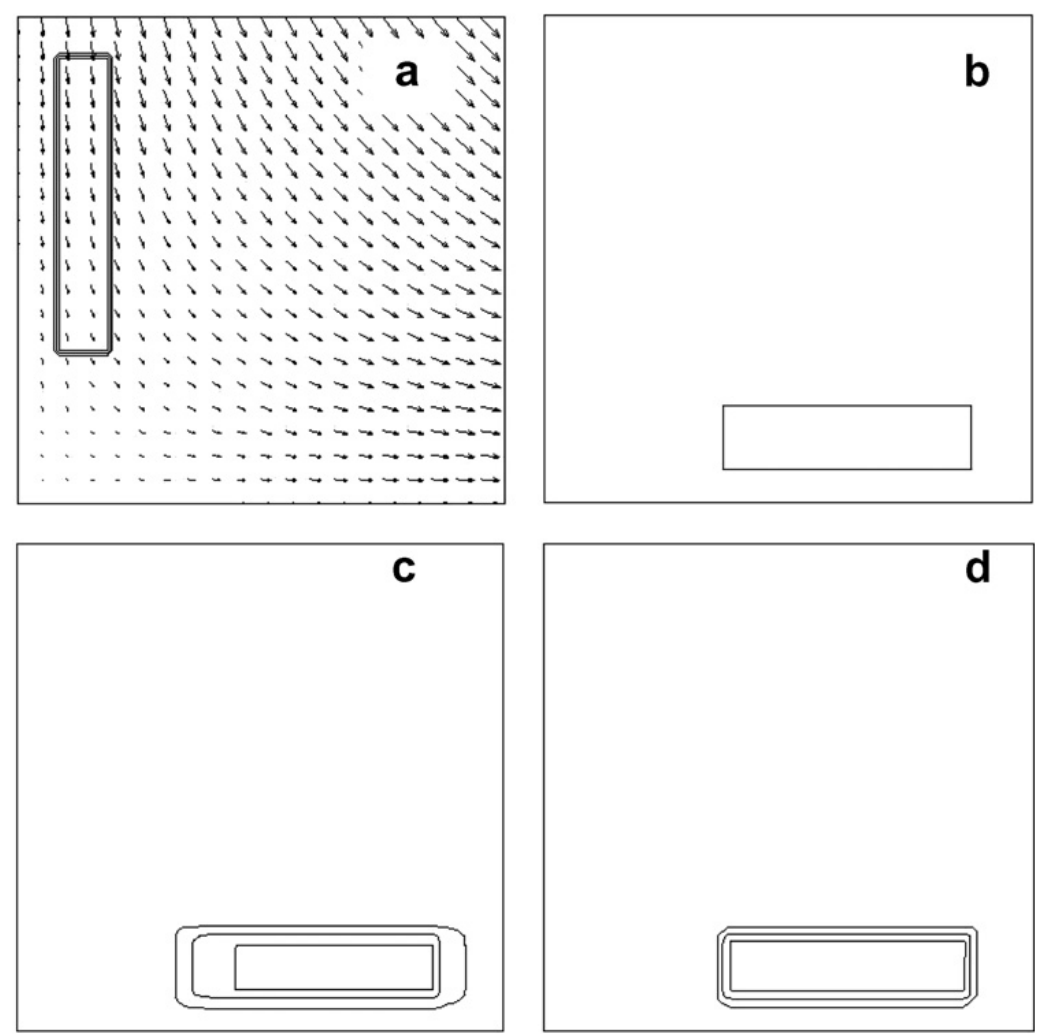

Fig. 9. Stretching-compression of a rectangle in a hyperbolic flow (iso- $C=0.01,0.5,0.99,100 \times 100$ uniform grid). (a) Initial condition; (b) theoretical solution; (c) solution obtained using $\boldsymbol{V}$; (d) solution obtained using $\widetilde{\boldsymbol{V}}$.

\subsection{Two-dimensional bubbles}

We now consider examples in which the whole set of governing equations (1)-(4) is solved and focus on the evolution of the computed interfacial thickness and mass of each fluid. The various strategies mentioned in Section 3.2, which differ by the manner the direction used to find the reference point $\boldsymbol{x}_{0}$ is selected, are considered. Here our goal is to show how this choice impacts the final result.

Computations are performed within a two-dimensional $(x, z)$ domain $3.75 D \times 2 D$ large $(D$ being the initial bubble diameter). We use a $150 \times 80$ grid size with a regular spacing in both directions $(\Delta x / D=\Delta z /$ $D=2.5 \times 10^{-2}$ ). Free-slip boundary conditions are imposed on lateral boundaries while periodic conditions are imposed on the top and bottom boundaries. The density and viscosity ratios are set to $10^{3}$ and $10^{2}$ respectively, and the physical properties are chosen so that the Morton (or Kapitza) number is $M o=g \mu_{l}^{4} / \rho_{l} \sigma^{3}=5.3 \times 10^{-6}$ and the Bond (or Eötvos) number is $B o=\rho_{1} g D^{2} / \sigma=7.3$. In the above numbers, $\rho_{1}$ and $\mu_{1}$ are the density and viscosity of the liquid, respectively, $\sigma$ is the surface tension and $g$ denotes gravity. The final Reynolds number is expected to be of $\mathrm{O}\left(10^{2}\right)$.

Fig. 10 shows the evolution of the computed bubble obtained with four different variants of the transport algorithm. Note that Case $d$ corresponds to our final strategy. When the standard algorithm corresponding to Case a is used, the interface smears quite rapidly and the iso-contours of the volume fraction at the rear of the bubble spread out in the whole computational domain. Using the modified velocity $\widetilde{\boldsymbol{V}}$ (Cases b-d) maintains the interface much sharper and forces the bubble trajectory to evolve from a rectilinear path at early times to a zigzag path. However, a significant smearing remains at the rear of the bubble in Cases b and c, i.e. in regions where the interface is strongly curved. In contrast, there is almost no smearing in Case d, where streamlines and grid lines are both used to find $x_{0}$. This example shows in particular that the mixed strategy we finally selected is more efficient than a strategy in which $\boldsymbol{x}_{0}$ is seeked along the direction of $\nabla C$ as in Case c.

Fig. 11 compares the evolution of the mass error in Cases a (where no mass correction is performed) and $d$ in which $\Delta m_{\max }=10^{-5}$ is imposed in (8). The mass error clearly oscillates within the imposed bounds in the latter 
a

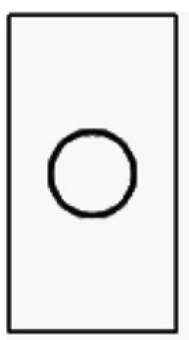

b

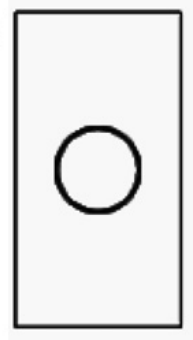

C

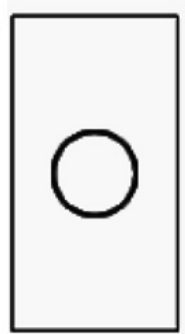

d

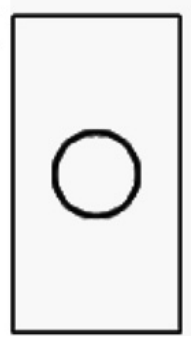

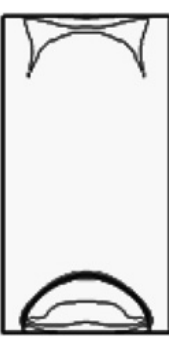
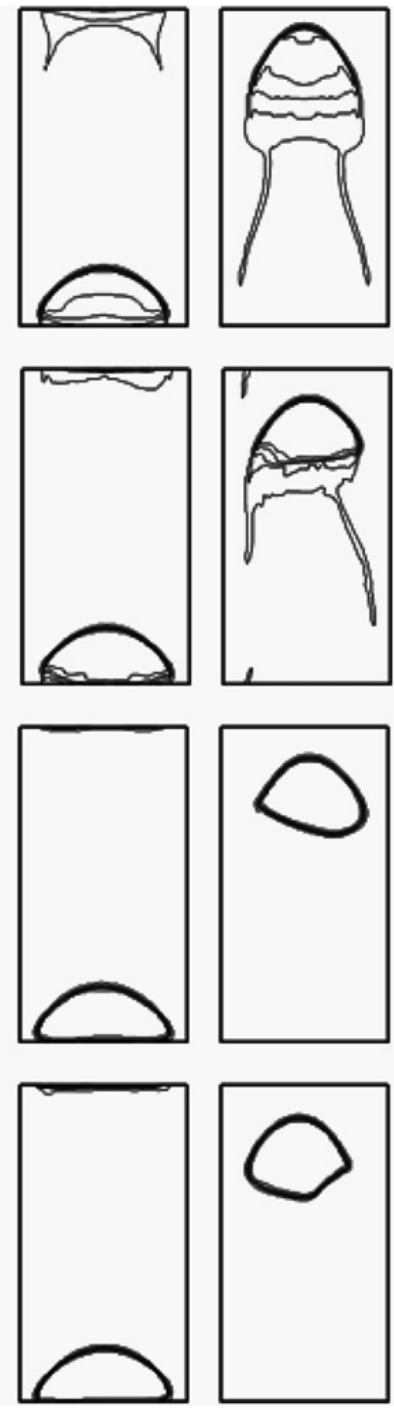
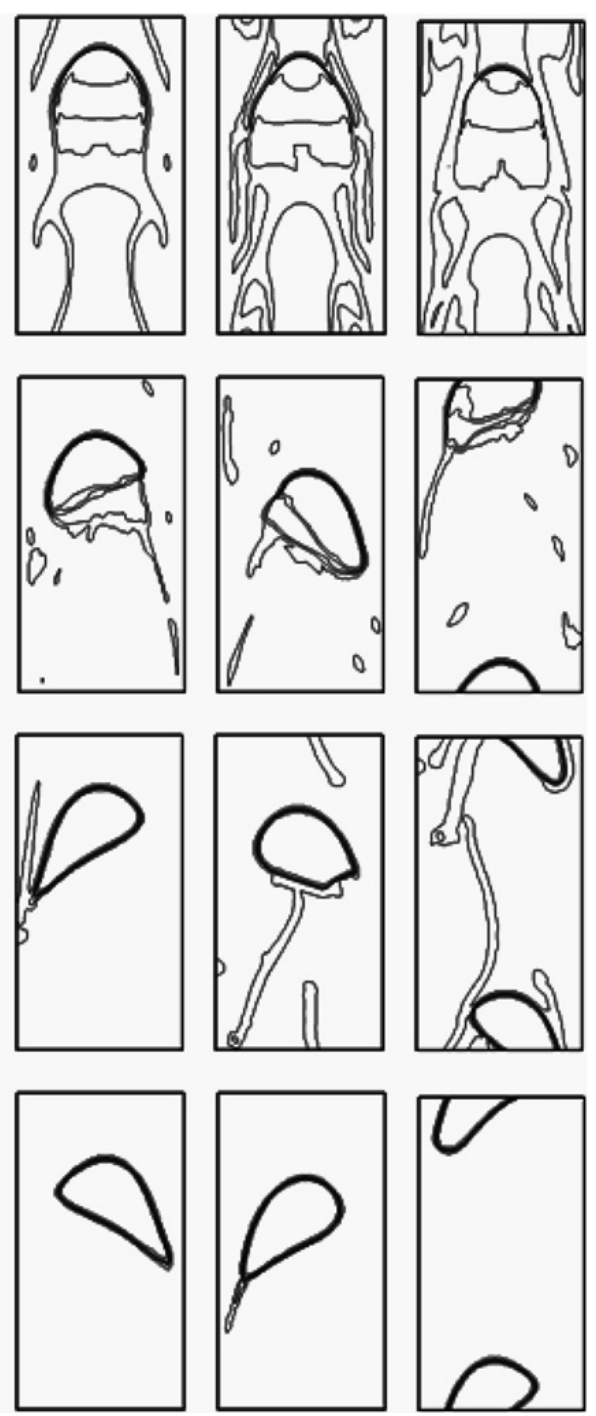

Fig. 10. A two-dimensional gas bubble rising in a low-viscosity liquid $\left(\rho_{1} / \rho_{\mathrm{g}}=10^{3}, \mu_{1} / \mu_{\mathrm{g}}=10^{2}, M o=5.3 \times 10^{-6}, B o=7.3\right)$. The dimensionless time step $\Delta t(g / D)^{1 / 2}$ between successive snapshots is 6.2 . Iso- $C=0.01,0.25,0.5,0.75,0.99$. (a) Standard technique $(\widetilde{\boldsymbol{V}}=\boldsymbol{V}$, no control of mass errors); (b) $x_{0}$ seeked using grid lines; (c) $x_{0}$ seeked using the direction of $\nabla C$; (d) $x_{0}$ seeked using the complete procedure involving both streamlines and grid lines. Note that mass error is controlled in cases (b)-(d).

case. In contrast, when the mass control procedure is switched off, the mass error grows until it reaches a value of about $9 \times 10^{-3}$. While this value is still quite acceptable in the present case, it may be one order of magnitude larger in other situations, so that the mass error really needs to be controlled to guarantee sound results.

\subsection{Spatial convergence of full Navier-Stokes solutions}

As we saw above, the present method inherently implies a transition region whose typical thickness is about three computational cells. Thus we can wonder how the spatial resolution affects the dynamics of a real twophase flow. To illustrate this point we report on two examples in which we computed the evolution of a deformable bubble using several grid spacings.

Our first example is concerned with small-amplitude shape oscillations of a gas bubble immersed in a liquid. As is well known, this problem has an analytical solution, making it convenient to perform a precise convergence study. We select density and viscosity ratios of $10^{3}$ and $10^{2}$, respectively, and an Ohnesorge number $O h=\left(\rho_{1} D \sigma / 2\right)^{1 / 2} / \mu_{1}$ of $10^{2}$, so that viscosity has a negligible influence on the short-time dynamics of the oscillations. The initial shape of the bubble is a prolate ellipsoid with a major axis $D_{\mathrm{M}}=(1+\varepsilon) D$ and two minor 

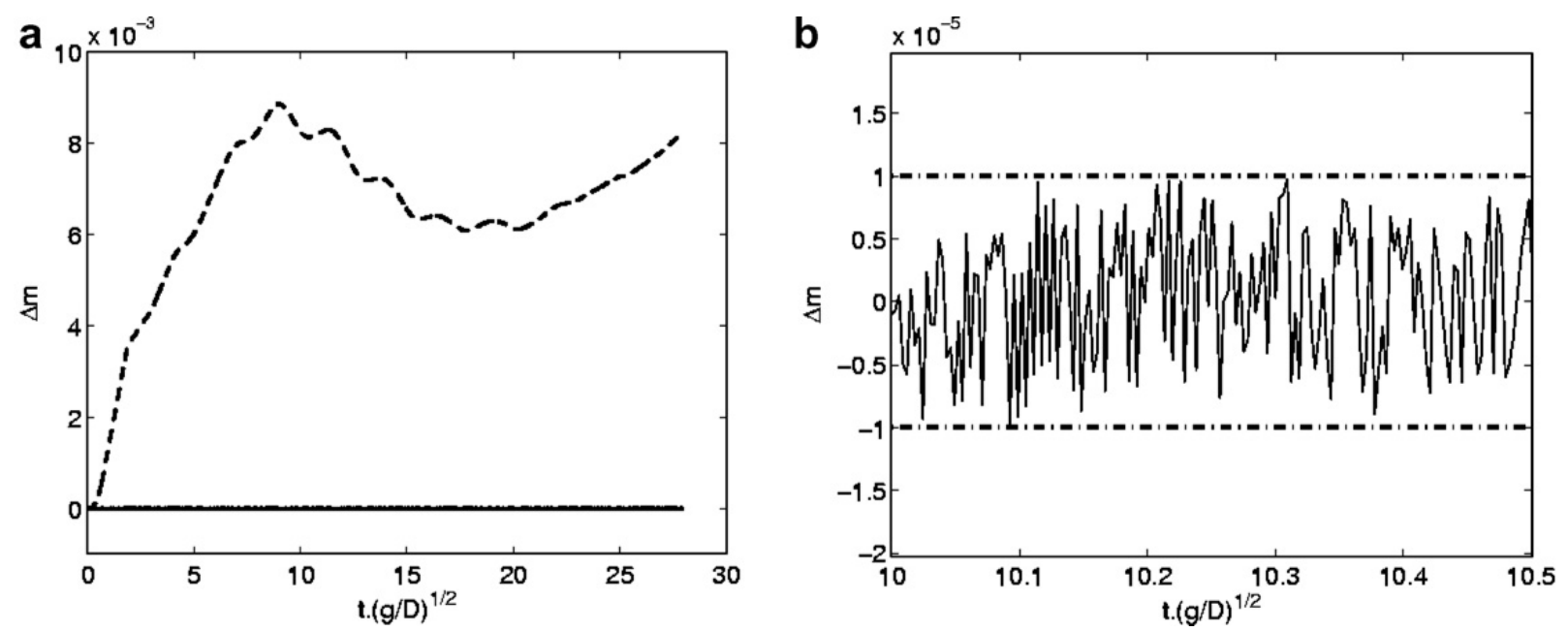

Fig. 11. (a) Evolution of the mass error in the computations of Fig. 10(a) (dashed line) and 10(d) (solid line). (b) Close up of the evolution of the mass error in the computations of Fig. 10(d) (the dotted lines correspond to the criterion $\left|\Delta m_{\max }\right|=10^{-5}$ used in Eq. (8)).

axes $D_{\mathrm{m}}=D(1+\varepsilon)^{-1 / 2}$, with $\varepsilon=0.05$. It is well known that in the small-amplitude regime, the radian frequency $\omega_{n}$ of the $n$th mode obeys the linear dispersion relation (Lamb, 1945)

$$
\omega_{n}^{2}=(n+1)(n-1)(n+2) \frac{8 \sigma}{\rho_{l} D^{3}}
$$

Computations are performed within a cylindrical $(r, z)$ domain $2.5 D \times 5 D$ large. Free-slip boundary conditions are imposed on all boundaries. The computation is stopped after $8 \tau, \tau$ being the capillary time defined through $\tau=\left(\rho_{1} D^{3} / 8 \sigma\right)^{1 / 2}$. The frequency is obtained by monitoring the instantaneous aspect ratio of the bubble and performing its Fourier transform. We perform this test on grids of increasing resolution ranging from $50 \times 100$ to $400 \times 800$. The results are summarized in Fig. 12. A regular convergence towards the theoretical frequency is observed as the grid is refined. The order of convergence is found to be about 1.5 as shown by the solid line in Fig. 12. The present test indicates that the capillary force is satisfactorily evaluated and is correctly coupled with the other contributions involved in the Navier-Stokes equations.

The second test concerns a deformable bubble rising in a liquid at rest. The dimensionless parameters are $\rho_{\mathrm{l}} / \rho_{\mathrm{g}}=10^{3}, \mu_{\mathrm{l}} / \mu_{\mathrm{g}}=10^{2}, M o=10^{-2}$ and $B o=10$. The cylindrical computational domain is now discretized with a uniform grid whose spacing ranges from $\Delta x=D / 10$ to $\Delta x=D / 90$. Figs. 13 and 14 show how the rise velocity (computed using Eq. (11) below) and final shape of the bubble change with the spatial discretization. Fig. 13 reveals that the difference between the terminal velocity corresponding to $\Delta x=D / 20$ and that obtained with $\Delta x=D / 90$ is less than $5 \%$ and the curves $V=f(t)$ converge as the resolution increases; in particular there

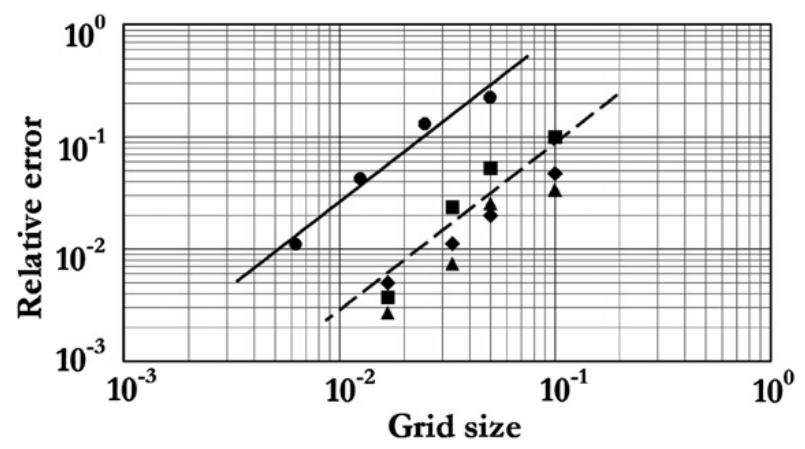

Fig. 12. Evolution of the computational error with the grid size in some bubble computations. Rising bubble of Fig. 13: ( $\mathbf{\square})$ Terminal velocity $V ;(\downarrow)$ length of the minor axis $d ;(\boldsymbol{\Delta})$ length of the major axis $D$ (the reference is the result obtained with the finest grid). ( Frequency of shape oscillations for the oscillating bubble in Section 4.3 (the reference is the theoretical prediction). The grid size is made non-dimensional by dividing it with $D$. The slope of both lines is 1.5 . 


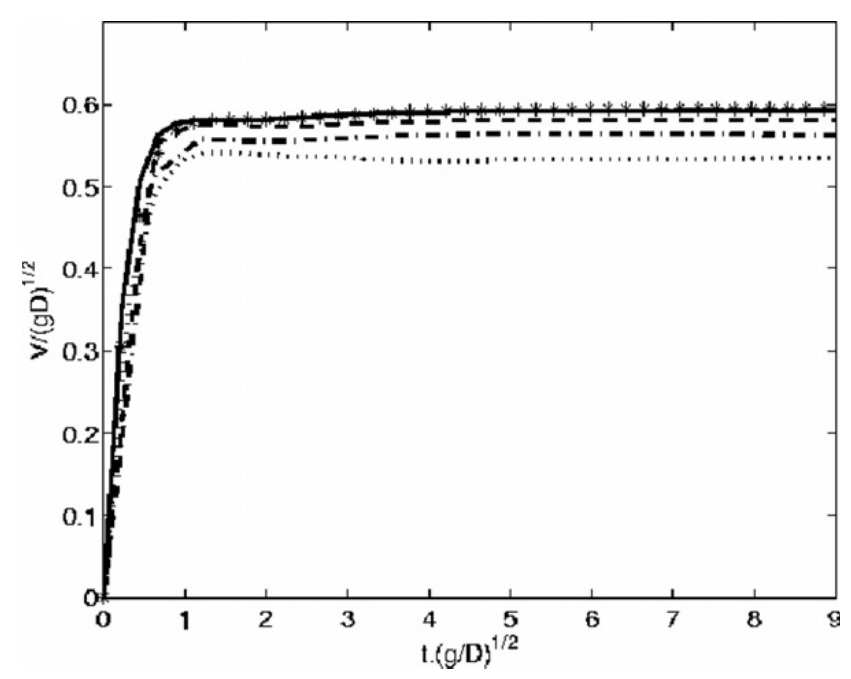

Fig. 13. Time evolution of the bubble rise velocity. (...) $\Delta x=D / 10 ;\left(--^{-*-\cdot)} \Delta x=D / 20 ;(----) \Delta x=D / 30 ;(-) \Delta x=D / 60 ;\left({ }^{* * * *}\right)\right.$ $\Delta x=D / 90\left(B o=10, M o=10^{-2}\right)$.
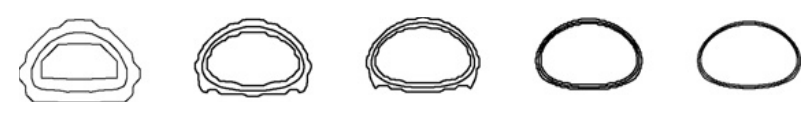

Fig. 14. Final shape of the bubble (same physical parameters as in Fig. 13). From left to right: $\Delta x=D / 10$ to $\Delta x=D / 90$ (iso-C $=0.01,0.5$ and 0.99 ).

is almost no difference between the two curves obtained with $\Delta x=D / 60$ and $\Delta x=D / 90$, respectively. According to Fig. 14, the shape of the bubble exhibits little sensitivity to the spatial resolution, except in the case $\Delta x=D / 10$ which is clearly under-resolved, and almost no change is discernible between the various resolutions where $\Delta x$ is smaller than $D / 30$. These results suggest that in this range of parameters, the present numerical approach correctly captures bubble dynamics even with modest resolutions, typically 20-30 cells per diameter (obviously this lower bound increases with the Reynolds and Bond number). A quantitative analysis of the sensitivity to the grid is provided in Fig. 12 where three indicators are considered, namely the terminal bubble velocity $V$, the length of the minor axis $d$ and that of the major diameter $D$, the latter two being defined by locating the position of the isovalue $C=0.5$. The relative error on each of these indicators is defined by considering the result obtained on the finest grid $(\Delta x=D / 90)$ as the exact solution. Again, the order of convergence is found to be about 1.5 as shown by the dashed line in Fig. 12.

\section{Some aspects of bubble dynamics}

We now leave numerical tests and turn toward several situations of physical interest involving gas bubbles moving in an incompressible liquid. Most computations discussed in this section are axisymmetric but some of them are fully three-dimensional. Since the density and viscosity ratios are large, gas bubbles rising in a Newtonian liquid are usually characterized by three dimensionless parameters, namely the Reynolds number $R e=\rho_{1} V D / \mu_{1}$, the Bond number $B o$ and the Morton $M o$ (see Section 4.2 for definitions). While the Bond and Morton numbers may be selected a priori by the experimentalist, the Reynolds number is a result of the bubble dynamics. The rise velocity $V$ of the bubble is defined as its barycentric velocity in the vertical direction, viz.

$$
V(t)=\frac{\int_{\vartheta} C(\boldsymbol{x}, t) \boldsymbol{V}(\boldsymbol{x}, t) \cdot \boldsymbol{e}_{z} \mathrm{~d} \vartheta}{\int_{\vartheta} C(\boldsymbol{x}, t) \mathrm{d} \vartheta}
$$

where $C$ is the gas volume fraction, $\boldsymbol{e}_{z}$ is the unit vector in the vertical direction and $\vartheta$ denotes the volume of the whole computational domain. 


\subsection{Buoyant bubbles: revisiting Clift, Grace and Weber's map}

As a first physically meaningful application, we now examine the evolution of gas bubbles rising under gravity over a wide range of Bond and Reynolds numbers, typically $B o=\mathrm{O}\left(1-10^{3}\right)$ and $R e=\mathrm{O}\left(1-10^{3}\right)$. Clift et al. (1978) plotted the experimentally observed shape of rising bubbles within this range in a diagram using the Bond and Reynolds number as axes, isovalues of the Morton number appearing in the back. This map is frequently used in the chemical engineering community. Here we revisit this map numerically and compare our results with those of the available literature. For this purpose, we perform a series of axisymmetric computations over a wide range of Morton number $\left(10^{-11}<M o<5 \times 10^{6}\right)$ and Bond number $\left(1<B o<10^{3}\right)$, leading a posteriori to Reynolds numbers $R e$ ranging from 1 to $10^{3}$, approximately. Therefore the Morton number ranges from values corresponding to extremely viscous oils to water. Similarly, the Bond number ranges from values at which the capillary pressure $\sigma / D$ may balance the hydrostatic head $\rho_{1} g D$ to values at which the former is only a very small fraction of the latter. Note that when the Reynolds number exceeds some hundreds, real bubbles may not remain axisymmetric, owing to wake instability (Magnaudet and Eames, 2000), so that the axisymmetric results presented below must be regarded with some caution in this range.

The computations are performed within a cylindrical $(r, z)$ domain $5.5 D \times 12.6 D$ large. We use a $140 \times 700$ grid size with a regular spacing in the $z$-direction $\left(\Delta z / D=1.8 \times 10^{-2}\right)$. In the $r$-direction, the spacing is regular over a central zone $0 \leqslant r \leqslant 1.8 D$ corresponding to the region crossed by the bubble and that of maximum liquid entrainment $\left(\Delta r / D=1.8 \times 10^{-2}\right)$. The outer region $1.8 D<r \leqslant 5.5 D$ is discretized with 40 cells whose size increases with $r$ following an arithmetic distribution. In the vertical direction, the grid is uniform, i.e. the grid spacing $\Delta z / D$ is about $1 / 55$. Free-slip boundary conditions are imposed on the top, bottom and lateral boundaries, so that the fluid entrained upward by the bubble slowly goes down near the lateral boundary; obviously computations are stopped before the bubble comes close to the upper boundary, to avoid contamination of the results by confinement effects. The density ratio $\rho_{1} / \rho_{\mathrm{g}}$ and the viscosity ratio $\mu_{\mathrm{l}} / \mu_{\mathrm{g}}$ are set to $10^{3}$ and $10^{2}$ respectively. All computations are started by releasing a spherical bubble from rest. The range of parameters covered in this investigation is summarized in Table 1.

Results exhibiting very contrasted shapes of bubbles are displayed in Fig. 15. Following the description of Bhaga and Weber (1981), we observe spherical bubbles (Case a), oblate ellipsoidal cap bubbles (Cases c and d), ellipsoidal bubbles (Case f), dimpled ellipsoidal cap bubbles (Case g), skirted bubbles (Case h), oblate ellipsoidal bubbles (Case j), toroidal bubbles (Cases k, 1 , o and $p$ ) and wobbling bubbles (Case $n$ ). The three iso-contours $C=0.01,0.5$ and 0.99 are almost superimposed in most cases. Some local smearing of the front may however be observed in cases where a pronounced dimple or skirt is present. Present results may be compared with those of Fig. 17 in which we reported the experimental observations of Bhaga and Weber (1981) and the numerical results of Unverdi and Tryggvason (1992) and Ryskin and Leal (1984) (note that in the latter reference the authors classified their results according to the value of the Reynolds number $R e$ and the Weber number $W e=\rho_{1} V^{2} D / \sigma$ ). The bubble shapes of Fig. 15 corresponding to Cases $\mathrm{c}, \mathrm{f}, \mathrm{i}, \mathrm{j}$ are in good agreement with those obtained in Ryskin and Leal (1984) through a boundary-fitted technique (Cases $\varepsilon, \phi, \gamma$ and $\eta$ in Fig. 17). A good correspondence is also observed with the experiments of Bhaga and Weber (1981), even though the corresponding Bond and Reynolds numbers were slightly different from those of Table 1 (Cases c, $\mathrm{g}$ and $\mathrm{h}$ in Fig. 15 and $\alpha, \beta$ and $\chi$ in Fig. 17). Finally the comparison with the computations of Unverdi and Tryggvason (1992) reveals a good agreement at low-to-moderate $B o$ (Cases e, $\mathrm{f}$ and $\mathrm{i}$ in Fig. 15 and $l, \varphi$ and $\lambda$ in Fig. 17) but shows slight differences at the rear part of the bubble for

Table 1

Dimensionless parameters of the computations corresponding to Fig. 15 (the approximate value of the Reynolds number is calculated a posteriori)

\begin{tabular}{|c|c|c|c|c|}
\hline Morton number & $B o=1$ & $B o=10$ & $B o=10^{2}$ & $B o=10^{3}$ \\
\hline$R e \approx 1$ & (a) $5 \times 10^{-3}$ & (b) 5 & (c) $5 \times 10^{3}$ & (d) $5 \times 10^{6}$ \\
\hline$R e \approx 10$ & (e) $10^{-5}$ & (f) $10^{-2}$ & (g) 10 & (h) $10^{4}$ \\
\hline$R e \approx 10^{2}$ & (i) $5 \times 10^{-8}$ & (j) $5 \times 10^{-6}$ & (k) $10^{-3}$ & (1) 1 \\
\hline $\operatorname{Re} \approx 10^{3}$ & (m) $10^{-11}$ & (n) $10^{-9}$ & (o) $10^{-7}$ & (p) $10^{-4}$ \\
\hline
\end{tabular}




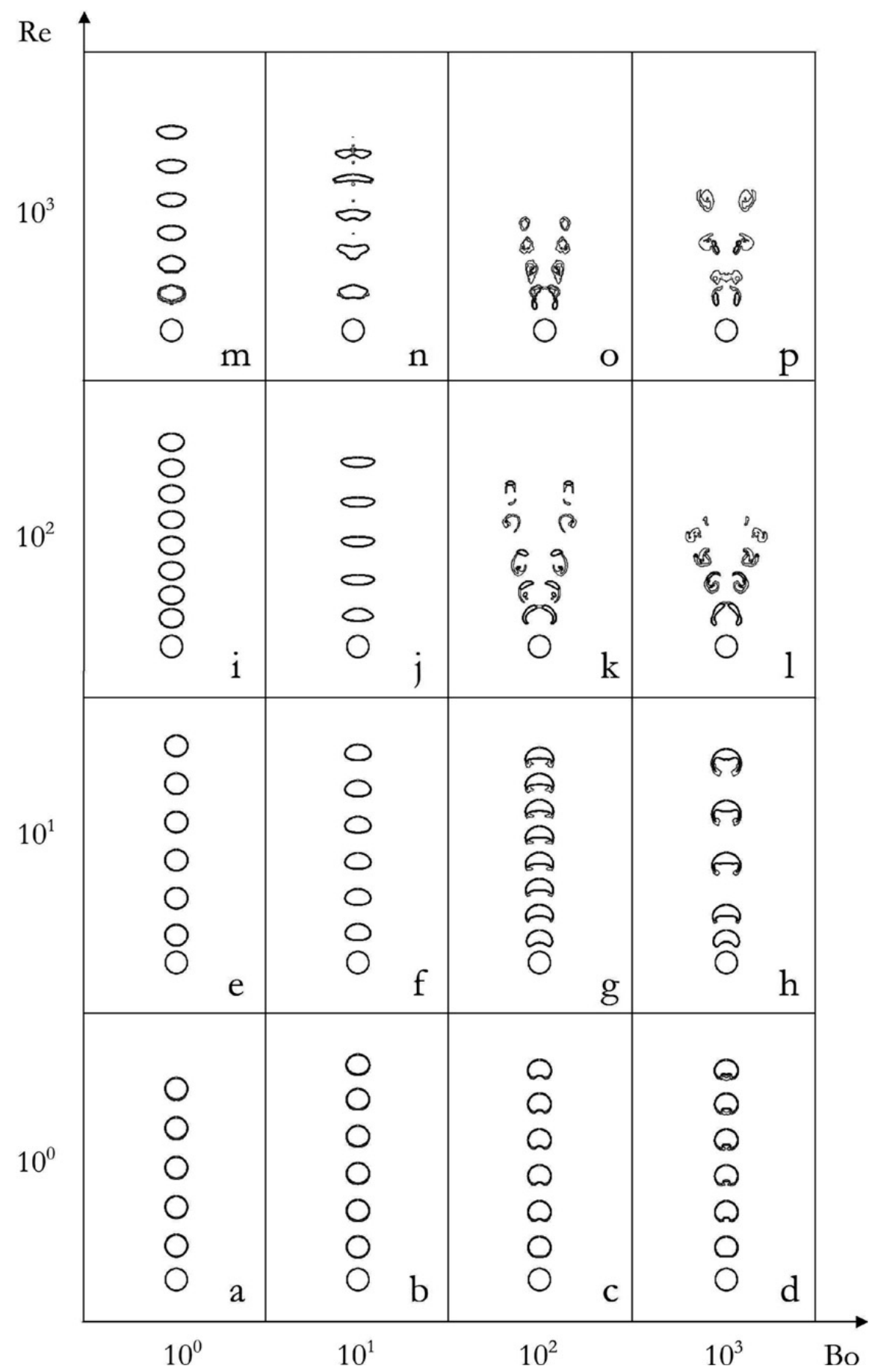

Fig. 15. Buoyant bubbles rising in a Newtonian liquid $\left(\rho_{1} / \rho_{\mathrm{g}}=10^{3}, \mu_{\mathrm{l}} / \mu_{\mathrm{g}}=10^{2}, \Delta x / D=1 / 60\right.$, iso-C $=0.01,0.5$ and 0.99$)$.

$B o=\mathrm{O}\left(10^{2}\right)$ and $R e=\mathrm{O}(10)$. In this range of parameters, our computations result in a bubble exhibiting a short dimple, whereas a skirted bubble was observed in Unverdi and Tryggvason (1992) (Case g in Fig. 15 and $\kappa$ in Fig. 17). It may be that, owing to the limitation of the present method in the capture of thin filaments, the thin skirt predicted by these authors cannot be reproduced; on the other hand, the shape we observe is very similar to that revealed by the photograph of Bhaga and Weber (1981) in the same range of parameters (Case $\beta$ in Fig. 17). Note that when Re is large while $B o$ is of $\mathrm{O}(10)$ (Cases $\mathrm{j}$ and $\mathrm{n}$ in Fig. 15), shape oscillations occur during the rise of the bubble. Finally, when inertia effects are large while capillary effects are very small (Cases 1 , o and p), our computations indicate that the bubble becomes toroidal. Such evolution has already been 
reported by Sussman and Smereka (1997) and Chen et al. (1999) who observed it for Bond and Reynolds numbers typically larger than $10^{2}$. Note that with this resolution, the computed bubble in the Case k in Fig. 15 is toroidal whereas the one reported by Bhaga and Weber (1981) (Case $\delta$ in Fig. 17) has a spherical-capped shape. A detail analysis of the evolution of the bubble topology in this range of parameters was performed by Bonometti and Magnaudet (2006) who showed that the spatial resolution plays a crucial role in the evolution of this topology and that a minimum of one hundred grid points per bubble diameter is required to obtain grid-independent results. In view of this, the present grid is not fine enough to provide accurate results in this very sensitive range. The reader is referred to the above paper for more details on the formation of toroidal bubbles.

The evolution of the Reynolds number Re of bubbles depicted in Fig. 15 is displayed in Fig. 16. These curves can be divided in two groups, with Group 2 including Cases k, 1, o and p of Fig. 15. Bubbles from Group 1 accelerate during a time period of $\mathrm{O}(\sqrt{g / D})$ and then reach a steady state Reynolds number. Case $\mathrm{n}$ in Figs. 15 and 16 exhibits periodic fluctuations about the mean value $R e=800$. These fluctuations are related to shape oscillations (Norman and Miksis, 2005) already observed experimentally (Clift et al., 1978; Stewart, 1995). Again, care has to be taken in the interpretation of this result as the path of real bubbles may not remain straight in this high- $R e$ regime. The second group is made of toroidal bubbles. The corresponding evolution of the Reynolds number is characterized by sharp peaks at early times $(t \sqrt{g / D}<3)$, followed by a strong decrease. For instance, $R e$ is reduced by more than $50 \%$ between $t \sqrt{g / D}=3$ and $t \sqrt{g / D}=15$ in Case k of Fig. 16. This decrease is accompanied by velocity fluctuations; the higher $R e$, the larger the amplitude of these fluctuations.

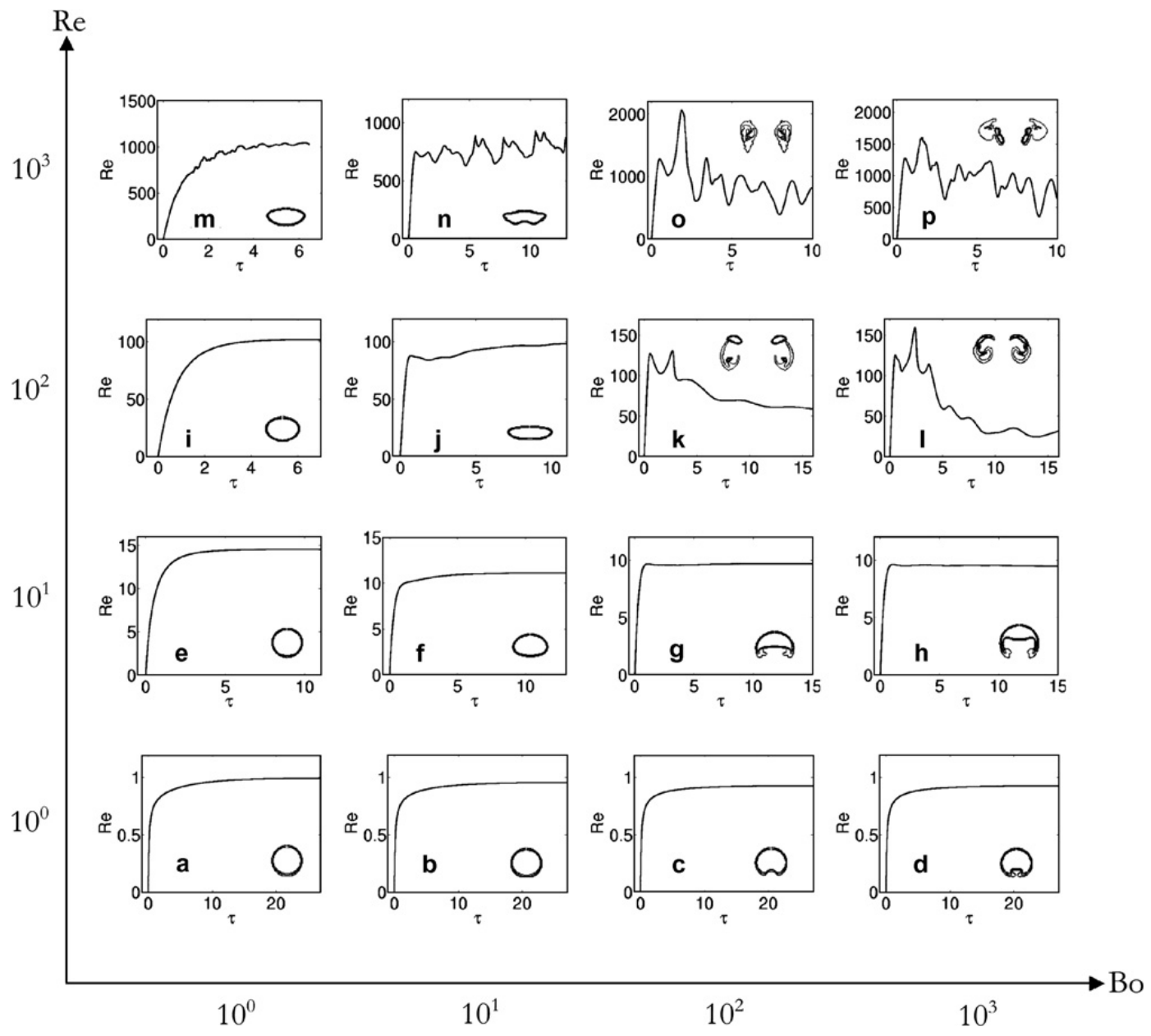

Fig. 16. Time evolution of the Reynolds number of the bubbles of Fig. 15. 


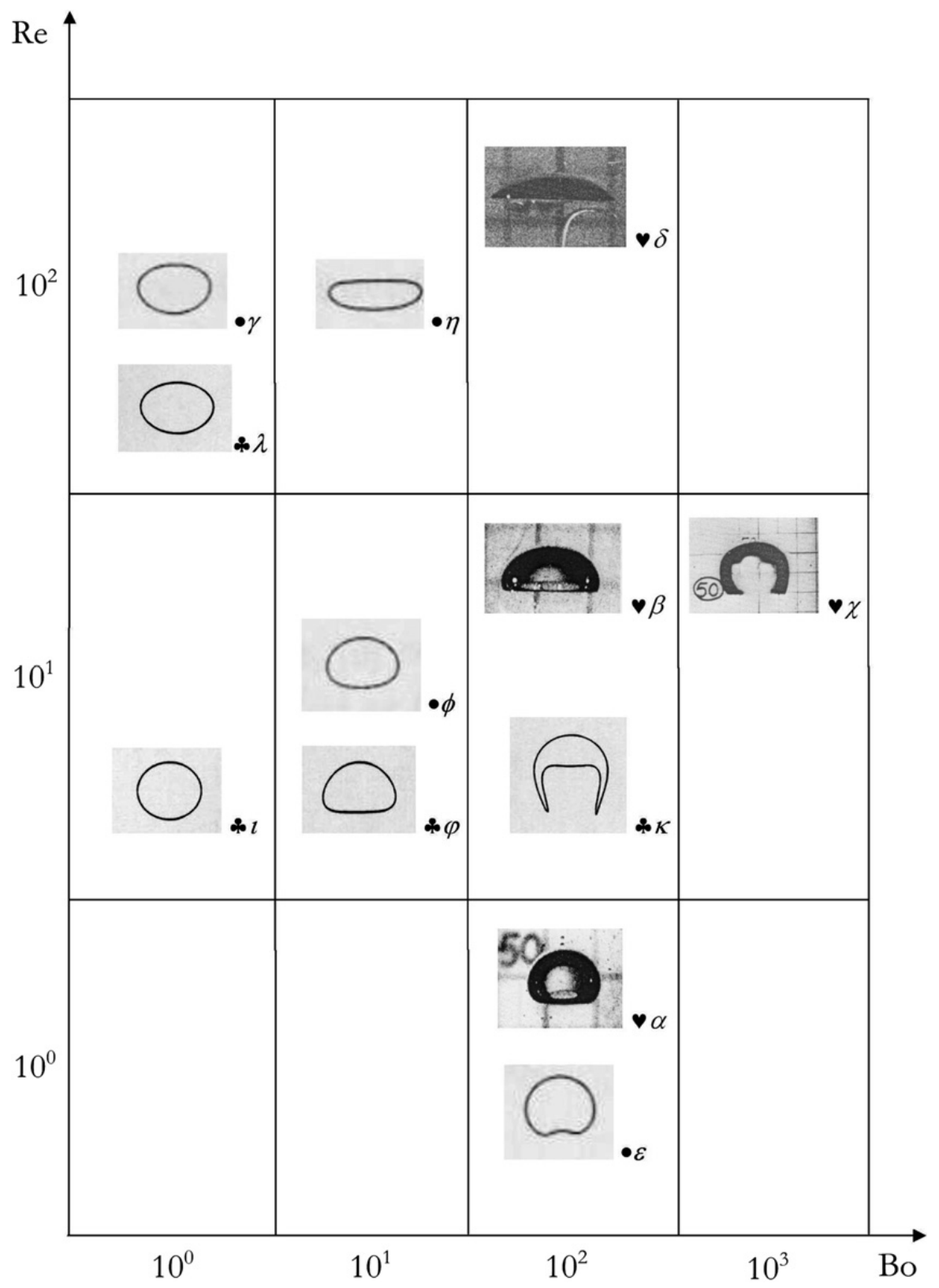

Fig. 17. Buoyant bubbles rising in a Newtonian liquid. Results from $(\vee)$ Bhaga and Weber (1981) with $\rho_{1} / \rho_{\mathrm{g}} \approx 1050$ and $\mu_{1} / \mu_{\mathrm{g}} \geqslant 4 \times 10^{3}(\alpha$ : $B o=116, \quad M o=848, \quad \operatorname{Re}=2.47 ;(\beta): B o=116, \quad M o=5.51, \quad \operatorname{Re}=13.3 ; \quad(\chi): \quad B o=339, \quad M o=43.1, \quad \operatorname{Re}=18.3 ; \quad(\delta): \quad B o=115$, $\left.M o=4.6 \times 10^{-3}, \operatorname{Re}=94\right) ;(\bullet)$ Ryskin and Leal (1984) with $\rho_{1} / \rho_{\mathrm{g}}=\infty$ and $\mu_{1} / \mu_{\mathrm{g}}=\infty(\varepsilon: \operatorname{Re}=1, W e=4 ;(\phi): R e=10, W e=4 ;(\gamma)$ : $\left.R e=10^{2}, W e=3 ;(\eta): R e=10^{2}, W e=8\right) ;(*)$ Unverdi and Tryggvason $(1992)$ with $\rho_{1} / \rho_{\mathrm{g}}=40\left(l: \mu_{1} / \mu_{\mathrm{g}}=277, B o=1, M o=10^{-5} ;(\varphi)\right.$ : $\left.\mu_{\mathrm{l}} / \mu_{\mathrm{g}}=277, B o=10, M o=10^{-2} ;(\kappa): \mu_{\mathrm{l}} / \mu_{\mathrm{g}}=269, B o=104, M o=10 ;(\lambda): \mu_{\mathrm{l}} / \mu_{\mathrm{g}}=88, B o=1, M o=10^{-7}\right)$.

\subsection{Motion of two bubbles rising in line}

We now consider the two-body interaction problem of nearly-spherical bubbles moving along their line of centres. This problem was addressed numerically by Yuan and Prosperetti (1994) for strictly spherical bubbles in the range $50 \leqslant R e \leqslant 200$, using a boundary-fitted method. They found that, based on the competition between the repelling irrotational (dipole) effect and the attractive suction of the trailing bubble in the wake of the leading one, the separation $H$ between the bubble centres reaches an equilibrium value which depends on the Reynolds number as $H / D=2.20 \log _{10} R e-2.19, D$ being the bubble diameter and $R e$ being based on the terminal bubble velocity. Their findings were mostly confirmed by Harper (1997) on the basis of an asymptotic study carried out in the limit $\operatorname{Re} \gg 1, H / D \rightarrow \infty$. A detailed experimental investigation of the same 
problem, combined with a modelling of the interaction process was reported by Katz and Meneveau (1996). They found that coalescence always occurs for $0.2 \leqslant R e \leqslant 35$, which suggests that the finite separation predicted by Yuan and Prosperetti (1994) for $R e>50$ may not exist at low-to-moderate Reynolds number. Here we make use of our computational technique to revisit this problem with slightly deformable bubbles by considering the two contrasted situations corresponding to $R e \approx 20$ and $R e \approx 180$.

The computations are performed on a grid similar to that used in Section 5.1. However, as the interaction process requires a fairly long time to reach equilibrium, the cylindrical $(r, z)$ domain is extended in the vertical direction and is chosen to be $8 D \times 24 D$ large. The grid size is $140 \times 1400$ and the region of regular spacing in the $r$-direction is $0 \leqslant r \leqslant 1.5 D$. Free-slip (resp. periodic) boundary conditions are imposed on the lateral (resp. horizontal) boundaries. The density and viscosity ratios are set to $10^{3}$ and $10^{2}$ respectively, whereas we select $B o=0.4$. In the first case (denoted as Case A) the Morton number is $M o=5 \times 10^{-7}$ while in the second case (Case B) we select $M o=1.75 \times 10^{-9}$. The two bubbles have the same initial diameter $D$ and are initially separated by a centre-to-centre distance $H_{0}=2.5 \mathrm{D}$. The computation is started by releasing the two spherical bubbles from rest. Note that the mass correction (8) is applied to each bubble individually with $\Delta m_{\max }=10^{-5}$ to insure that the system dynamics are not altered by small mass differences.

Fig. 18(a) shows the evolution of the two bubbles in Case A. As they rise, bubbles get closer and closer to each other, owing to the shielding effect produced by the wake of the leading bubble which acts to reduce the drag of the trailing one. As this effect keeps on acting, the two bubbles eventually coalesce. The resulting bubble rapidly takes a slightly oblate shape. Fig. 19(a) shows the evolution of the two bubble Reynolds numbers. The relative velocity first increases $\left(3<t(g / D)^{1 / 2}<9\right)$ so that the velocity of the trailing bubble is about $14 \%$ higher than that of the leading one at $t(g / D)^{1 / 2}=9$. This relative velocity then slightly decreases as the distance between the two bubbles becomes less than one diameter $\left(9<t(g / D)^{1 / 2}<14\right)$. Coalescence occurs at $t(g / D)^{1 / 2}=14$ and is accompanied by strong fluctuations of the velocity due to shape oscillations $\left(14<t(g / D)^{1 / 2}<17\right)$. The resulting bubble eventually reaches a steady Reynolds number about 23 . These results are in qualitative agreement with those of Katz and Meneveau (1996) who always observed coalescence in this range of Reynolds number. Note also that the empirical expression found by Yuan and Prosperetti (1994) for the final separation $H / D$ predicts values less than unity for $R e<28$, which suggests that the two bubbles should eventually overlap even though they were perfectly spherical.

The evolution of the two bubbles in Case B displayed in Fig. 18(b) is quite different. The leading bubble first rises slightly faster than the trailing one (see Fig. $19(\mathrm{~b}), t(g / D)^{1 / 2}<3$ ) because the repelling irrotational effect acts first. As the wake of the leading bubble develops, the trailing bubble accelerates and goes faster than the leading one $\left(3<t(g / D)^{1 / 2}<9\right)$. An equilibrium between the two competing effects is eventually found and both
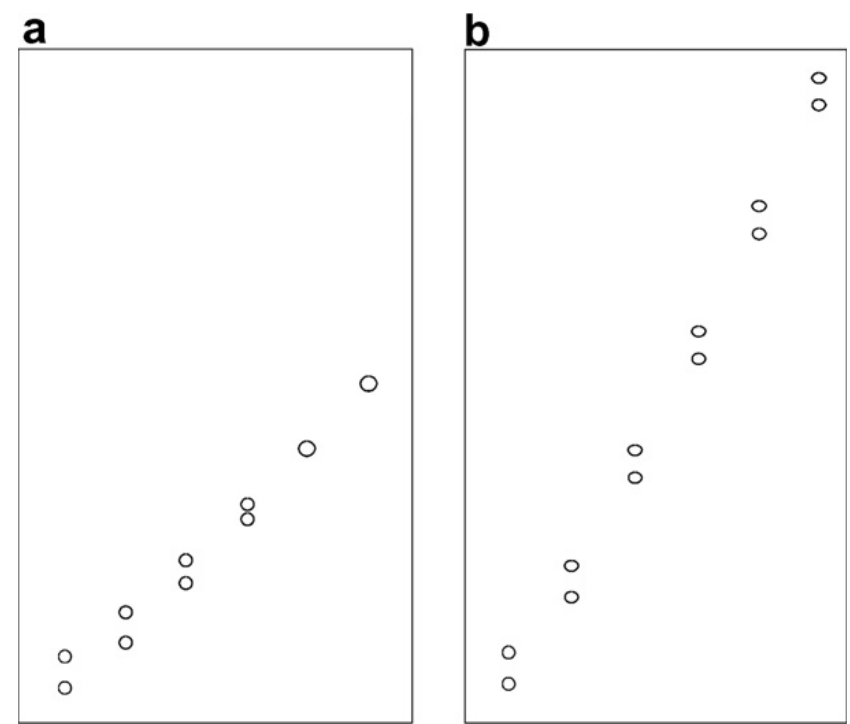

Fig. 18. Evolution of two nearly spherical bubbles rising in line $\left(\rho_{1} / \rho_{\mathrm{g}}=10^{3} ; \mu_{\mathrm{l}} / \mu_{\mathrm{g}}=10^{2} ; h / D=1.5, B o=0.4\right)$. (a) $M o=5 \times 10^{-7}$; (b) $M o=1.75 \times 10^{-9}$ (iso- $C=0.01,0.5$ and 0.99 ). The dimensionless time step $\Delta t(g / D)^{1 / 2}$ between successive snapshots is 4.4 . 
a

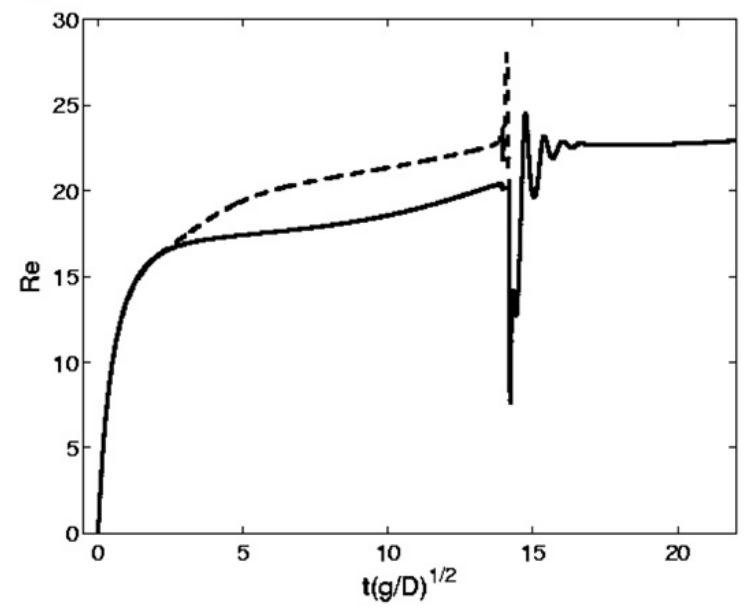

b

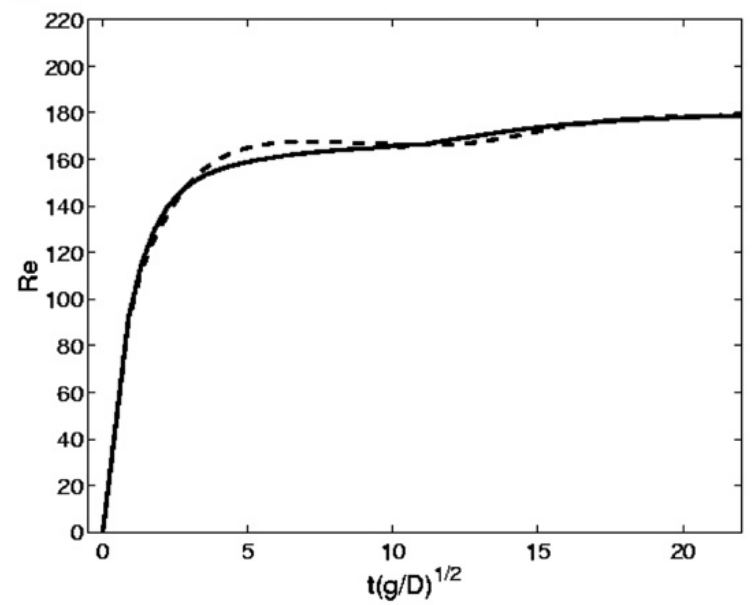

Fig. 19. Time history of the individual Reynolds numbers of the two bubbles of Fig. 18. (-) leading bubble; (----) trailing bubble. (a) $M o=5 \times 10^{-7}$; (b) $M o=1.75 \times 10^{-9}$.

bubbles reach the same final Reynolds number $R e \approx 180$. The equilibrium shape is displayed in Fig. 18(b). Both bubbles are slightly oblate, the aspect ratio of the leading (resp. trailing) bubble being $\chi_{1}=1.32$ (resp. $\left.\chi_{t}=1.21\right)$. It is known that a slightly deforming isolated bubble rising in the high-Reynolds number regime has an aspect ratio $\chi \approx 1+9 / 64 W e$, with $W e=\rho_{1} V^{2} D / \sigma$ (Moore, 1965). As $W e=R^{2}(M o / B o)^{1 / 2}$, we have $W e=2.1$ from which the above prediction yields $\chi=1.30$. The computed deformation of the leading bubble is close to this prediction, which was to be expected since the trailing bubble only slightly disturbs the flow around the leading bubble. In contrast the deformation of the trailing bubble is less than predicted for an isolated bubble because the pressure difference between its front stagnation point and its equator is smaller than that experienced by an isolated bubble, owing to the pressure reduction in the wake of the leading bubble. Fig. 18(b) indicates that the equilibrium separation is $H / D=2.23$, whereas Yuan and Prosperetti (1994) found $H / D=2.77$ for spherical bubbles with the same final Reynolds number. That the equilibrium separation found here with $B o=0.4$ is less than that corresponding to $B o=0$ indicates that the strength of the shielding effect increases more rapidly with the aspect ratio $\chi$ than the irrotational repelling effect.

\subsection{Interaction of two bubbles rising side by side}

We finally consider the problem of the lateral interaction between two nearly spherical bubbles rising side by side at moderate Reynolds number in a liquid at rest. This problem is of interest because it is now established, both experimentally (Takemura and Magnaudet, 2003) and numerically (Legendre et al., 2003), that the lateral force changes sign for a certain critical Reynolds number $R e_{0}$ of some tens. This force is repulsive (resp. attractive) when the bubble Reynolds number is smaller (resp. larger) than $R e_{0}$, a result of the competition between vortical and irrotational interaction mechanisms. For instance, Legendre et al. (2003) predicted that $R e_{0}$ is about 30 for two spherical bubbles whose centers are separated by a distance $h=1.5 D$ ( $D$ being the bubble diameter), a value confirmed by the experimental results of Takemura and Magnaudet (2003). As the lateral force is always a small percentage of the drag in these situations, it is challenging to see whether the present computational approach is capable of predicting the observed reversal of the lateral force.

For this purpose we consider two situations in which the density and viscosity ratios are set to $10^{3}$ and $10^{2}$ respectively, the Bond number is set to $B o=0.4$ and the initial separation $h$ between the two bubbles centers is $h / D=1.5$. In the first case (denoted as Case A) the Morton number is $10^{-6}$, so that $R e$ is about 10 , while in the second case (Case B) we select $M o=5 \times 10^{-8}$ so that $R e$ is about 50 . The grid extends over $9.5 D \times 8 D \times 4.5 D$ in the $(x, y, z)$ directions (see Fig. 20) and is made of $90 \times 60 \times 90$ cells. The cells are uniformly distributed, with $\Delta z / D=1 / 20$, along the vertical $(z)$ direction. In contrast, in the horizontal directions, the grid is only uniform within the central region crossed by the two bubbles (with $\Delta x / D=\Delta y /$ 
a

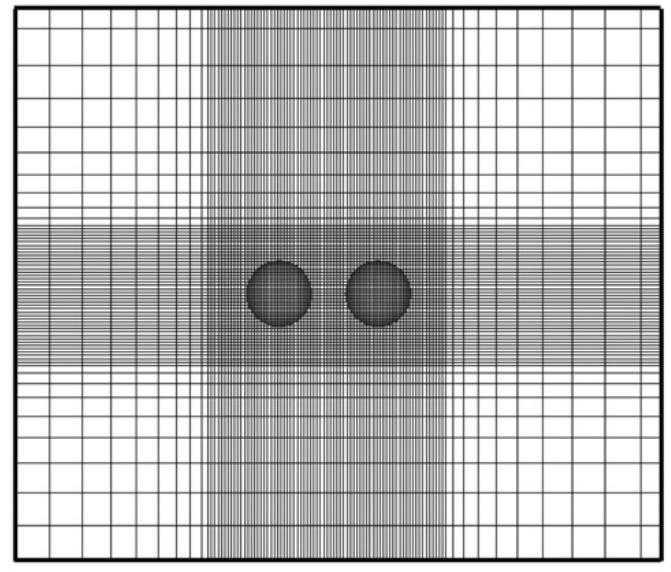

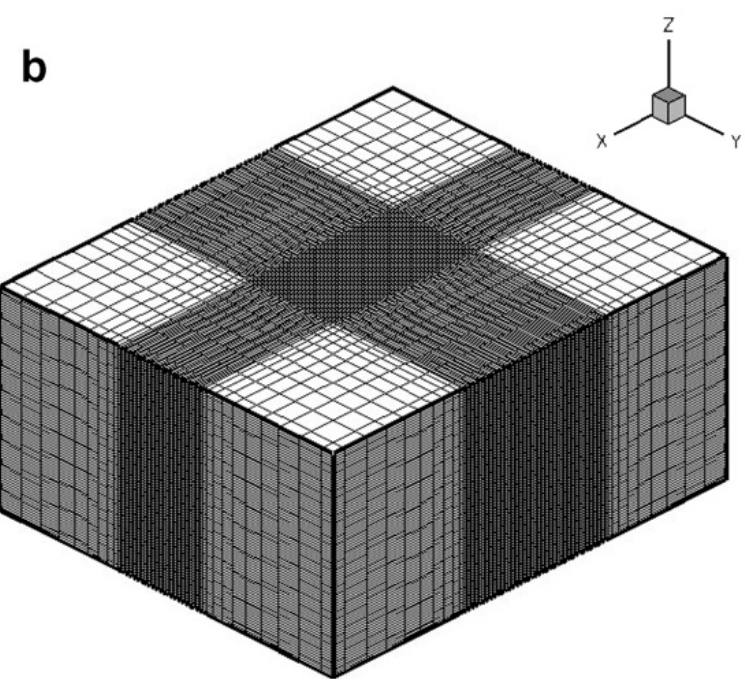

Fig. 20. The computational grid used in computations of Fig. 21. (a) Top view; (b) general view.

$D=1 / 20$ ), and is then nonuniform and coarser on the lateral part of the domain. Periodic conditions are imposed at the top and bottom boundaries while a free-slip condition is used on the vertical boundaries. The gap separating the two bubbles is initially described with ten cells and the two bubbles are released from rest. Note that, as we are using periodic conditions along the $z$-axis, we actually compute two chains of bubbles rising side by side rather than a single pair of bubbles. However in the flow regime considered here, the results of Yuan and Prosperetti (1994) indicate that this in-line interaction does not change the drag force by more than $5 \%$ for a vertical separation of $4.5 \mathrm{D}$. Thus, for the present purpose, the bubbles may be considered almost isolated in the vertical direction.

Fig. 21(a) shows how the two bubbles evolve in Case A. Clearly they slightly separate from each other as they rise, as predicted by Takemura and Magnaudet (2003) and Legendre et al. (2003). The deflection of the trajectory with respect to the vertical is about $4^{\circ}$. This deflection is small but this had to be expected on the basis of the relative value of the drag and transverse force for two strictly spherical bubbles. More precisely

a

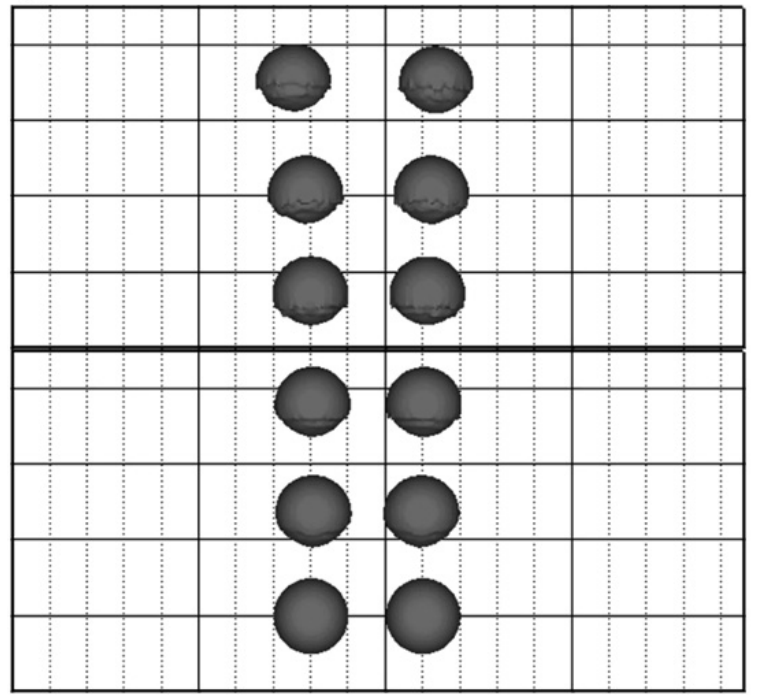

b

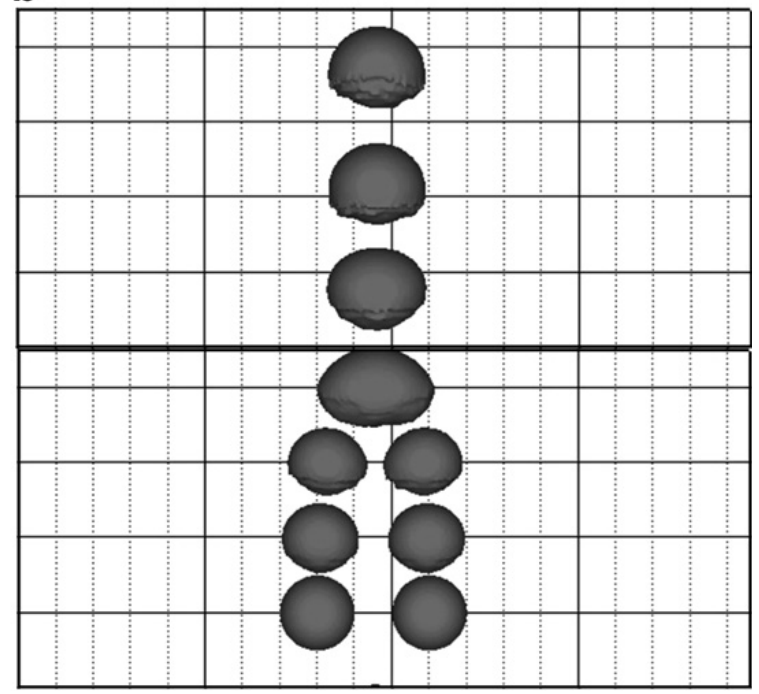

Fig. 21. Evolution of two nearly spherical bubbles rising side by side $\left(\rho_{1} / \rho_{\mathrm{g}}=10^{3} ; \mu_{1} / \mu_{\mathrm{g}}=10^{2} ; h / D=1.5, B o=0.4\right)$. (a) $M o=10^{-6}$; successive dimensionless times are $\tau=t(g / D)^{1 / 2}=0,2.46,4.42,6.39,8.36,10.82$; (b) $M o=5 \times 10^{-8}$; successive dimensionless times are $\tau=t(g / D)^{1 / 2}=0,1.47,2.46,3.41,4.39,5.38$ and 6.61 . (iso- $C=0.5$ ). 
Legendre et al. (2003) indicate that the drag coefficient is $C_{\mathrm{D}}=2.43$ for $R e=10$, whereas the "lift" coefficient is only $C_{\mathrm{L}}=0.15$ (both being defined by normalizing the corresponding component of the force with $\rho_{1} \pi D^{2} V^{2} / 8$ ). This yields a deflection angle of $3.5^{\circ}$ for $B o=0$, which compares well with the value of $4^{\circ}$ obtained for $B o=0.4$

The evolution is dramatically different in Case B (Fig. 21(b)). First, the separation between the two bubbles decreases, as expected from Takemura and Magnaudet (2003) and Legendre et al. (2003). Then the film separating the two bubbles breaks and the two bubbles coalesce, resulting in an oblate spheroidal bubble whose final Reynolds number is about 60. For strictly spherical bubbles, Legendre et al. (2003) predict that the final separation between the two bubbles should be $1.125 \mathrm{D}$. With the present grid, such a narrow gap is described by only 2 to 3 grid points, so that numerical coalescence can hardly be avoided. This phenomenon is reinforced by the smoothing procedure of the volume faction used in the computation of the capillary force (see Section 2) which makes this force act artificially through a layer with a finite thickness. Moreover, coalescence can physically be favored by the slight oblateness of the bubbles. A detailed study of the influence of the grid resolution and of the technique used to evaluate the capillary force during the late stages of the motion within the intersticial film is beyond the scope of the present work but remains an important issue for the future. At the present stage, we may at least conclude that, up to the accuracy allowed by the grid, Fig. 21 indicates that our code captures correctly the subtle hydrodynamic interactions between two bubbles rising side by side. In particular, it is able to predict correctly the sign reversal of the transverse force acting on the two bubbles.

\section{Conclusion}

We described a front-capturing method whose goal is to solve three-dimensional incompressible two-phase flows with large density and viscosity contrasts without involving any explicit interface reconstruction step, while maintaining sharp interfaces. The transported scalar quantity characterizing the two-phase nature of the flow is the volume fraction of one of the fluids. We developed simple correction strategies to conserve the mass of each fluid constant (since the volume fraction is transported in a non-conservative way), and to maintain the stiffness of the transition region surrounding the interface. For this purpose the volume fraction and velocity fields are suitably modified within the transition zone. In particular the velocity is made almost constant across this zone, so as to remove the spreading of the fronts in regions of stretching. Numerical tests demonstrated the ability of this correction strategy to properly transport the volume fraction in various types of flows; the accuracy of the overall method was also established on several examples dealing with bubble dynamics. A detailed study of several aspects of these dynamics was then carried out. In particular, the shape and velocity evolution of isolated bubbles were computed over a wide range of parameters and showed good agreement with available experimental results. The more subtle problem of head-on and side-byside interactions of two identical bubbles was then considered. In both cases, the corresponding results revealed that the numerical approach correctly reproduces the consequences of the competition between the vortical and the irrotational interaction mechanisms.

The next step of our work is to develop specific subgrid-scale models to take into account small-scale structures (such as small droplets and small bubbles) and subgrid-scale phenomena (such as the drainage of thin intersticial films) in the large-scale dynamics of complex bubbly flows.

\section{Acknowledgements}

This work was partly supported by a research grant from Arcelor Research whose help is very much appreciated. Some of the computational time was provided by the Scientific Groupment CALMIP where computations were run on Silicon Graphics Altix 3700 computers.

\section{References}

Adalsteinsson, D., Sethian, J.A., 1999. The fast construction of extension velocities in level set methods. J. Comput. Phys. 148, $2-22$. 
Benkenida, A., 1999. Développement et validation d'une méthode de simulation d'écoulements diphasiques sans reconstruction d'interface. Application à la dynamique des bulles de Taylor. Ph.D. Thesis. INP Toulouse.

Bhaga, D., Weber, M.E., 1981. Bubbles in viscous liquid: shapes, wakes and velocities. J. Fluid Mech. 105, 61-85.

Bonometti, T., 2005. Développement d'une méthode de simulation d'écoulements à bulles et à gouttes. Ph.D. Thesis. INP Toulouse.

Bonometti, T., Magnaudet, J., 2006. Transition from spherical cap to toroidal bubbles. Phys. Fluids 18, 052102.

Boris, J.P., Book, D.L., 1973. Flux-corrected transport: I. SHASTA, A fluid transport algorithm that works. J. Comput. Phys. 18, 248283.

Brackbill, J.U., Kothe, D.B., Zemach, C., 1992. A continuum method for modeling surface tension. J. Comput. Phys. 100, 335-354.

Bunner, B., Tryggvason, G., 2002. Dynamics of homogeneous bubbly flows. Part 1. Rise velocity and microstructure of the bubbles. J. Fluid Mech. 466, 17-52.

Calmet, I., Magnaudet, J., 1997. Large-eddy simulation of high-Schmidt-number mass transfer in a turbulent channel flow. Phys. Fluids 9 , 438-455.

Chang, Y.C., Hou, T.Y., Merriman, B., Osher, S., 1996. A level set formulation of Eulerian interface capturing methods for incompressible fluid flows. J. Comput. Phys. 124, 449-464.

Chen, L., Garimella, S.V., Reizes, J.A., Leonardi, E., 1999. The development of a bubble rising in a viscous liquid. J. Fluid Mech. 387, 6196.

Clift, R., Grace, J.R., Weber, M.E., 1978. Bubbles, Drops and Particles. Academic Press, New York.

Dendy, E.D., Padial-Collins, N.T., VanderHeyden, W.B., 2002. A general-purpose finite-volume advection scheme for continuous and discontinuous fields on unstructured grids. J. Comput. Phys. 180, 559-583.

De Sousa, F.S., Mangiavacchi, N., Nonato, L.G., Castelo, A., Tomé, M.F., Ferreira, V.G., Cuminato, J.A., McKee, S., 2004. A fronttracking/front-capturing method for the simulation of 3D multi-fluid flows with free surfaces. J. Comput. Phys. 198, 469-499.

Enright, D., Fedkiw, R.P., Ferziger, J., Mitchell, I., 2002. A hybrid particle-level set method for improved interface capturing. J. Comput. Phys. 183, 83-116.

Gomes, J., Faugeras, O., 2000. Reconciling distance functions and level sets. J. Vis. Commun. Image R. 11, $209-223$.

Harlow, F.H., Welch, J.E., 1965. Numerical calculation of time-dependent viscous incompressible flow of fluid with free surface. Phys Fluids 8, 2182-2189.

Harper, J.F., 1997. Bubbles rising in line: why is the first approximation so bad?. J. Fluid Mech. 351, 289-300.

Hirt, C.W., Nichols, B.D., 1981. Volume of Fluid (VOF) method for the dynamics of free boundaries. J. Comput. Phys. 39, $201-225$.

Juric, D., Tryggvason, G., 1998. Computation of boiling flows. Int. J. Multiphase Flow 24, 387-410.

Katz, J., Meneveau, C., 1996. Wake-induced relative motion of bubbles rising in line. Int. J. Multiphase Flow 22, $239-258$.

Lafaurie, B., Nardone, C., Scardovelli, R., Zaleski, S., Zanetti, G., 1994. Modelling merging and fragmentation in multiphase flows with SURFER. J. Comput. Phys. 113, 134-147.

Lamb, H., 1945. Hydrodynamics, sixth ed. Cambridge University Press.

Legendre, D., Magnaudet, J., 1998. The lift force on a spherical bubble in a viscous linear shear flow. J. Fluid Mech. 368, 81-126.

Legendre, D., Magnaudet, J., Mougin, G., 2003. Hydrodynamic interactions between two spherical bubbles rising side by side in a viscous liquid. J. Fluid Mech. 497, 133-166.

Magnaudet, J., Eames, I., 2000. The motion of high-Reynolds-number bubbles in inhomogeneous flow. Annu. Rev. Fluid Mech. 32, 659708.

Magnaudet, J., Rivero, M., Fabre, J., 1995. Accelerated flows past a rigid sphere or a spherical bubble. Part. I: Steady straining flow. J. Fluid Mech. 284, 97-135.

Moore, D.W., 1965. The velocity of rise of distorted gas bubbles in a liquid of small viscosity. J. Fluid Mech. 23, 749-766.

Norman, C.E., Miksis, J.M., 2005. Dynamics of a gas bubble rising in a inclined channel at finite Reynolds number. Phys. Fluids 17 , 022102 .

Osher, S., Sethian, J.A., 1988. Front propagating with curvature dependent speed: algorithms based on Hamilton-Jacobi formulation. J. Comput. Phys. 79, 12-49.

Popinet, S., Zaleski, S., 1999. A front tracking algorithm for accurate representation of surface tension. Int. J. Numer. Meth. Fluid 30, 775-793.

Renardy, M., Renardy, Y., Li, J., 2001. Numerical simulation of moving contact line problems using a volume of fluid method. J. Comput. Phys. 171, 243-263.

Rudman, M., 1997. Volume tracking methods for interfacial flow calculations. Int. J. Numer. Meth. Fluids 24, 671-691.

Rudman, M., 1998. A volume-tracking method for incompressible multifluid flows with large density variations. Int. J. Numer. Meth. Fluids 28, 357-378.

Ryskin, G., Leal, L.G., 1984. Numerical solution of free boundary problems in fluid mechanics. J. Fluid Mech. $148,1-43$.

Scardovelli, R., Zaleski, S., 1999. Direct numerical simulation of free-surface and interfacial flow. Annu. Rev. Fluid Mech. $31,567-603$.

Sethian, J., 1999. Level Set Methods and Fast Marching Methods. Cambridge University Press, Cambridge.

Shin, S., Juric, D., 2002. Modeling three-dimensional multiphase flow using a level contour reconstruction method for front tracking without connectivity. J. Comput. Phys. 180, 427-470.

Spelt, P.D.M., 2005. A level-set approach for simulations of flows with multiple moving contact lines with hysteresis. J. Comput. Phys. 207, 389-404.

Stewart, C.W., 1995. Bubble interaction in low-viscosity liquids. Int. J. Multiphase Flow 21, 1037-1046.

Sussman, M., Puckett, E.G., 2000. A coupled level set and volume-of-fluid method for computing 3D and axisymmetric incompressible two-phase flows. J. Comput. Phys. 162, 301-337. 
Sussman, M., Smereka, P., 1997. Axisymmetric free boundary problems. J. Fluid Mech. 341, 269-294.

Sussman, M., Uto, S., 1998. A computational study of the spreading of oil underneath a sheet of ice. CAM Report, 32-98.

Sussman, M., Smereka, P., Osher, S., 1994. A level set approach for computing solutions in incompressible two-phase flow. J. Comput. Phys. 114, 146-159.

Sussman, M., Fatemi, E., Smereka, P., Osher, S., 1998. An improved level set method for incompressible two-phase flows. Comput. Fluids 27, 663-680.

Takemura, F., Magnaudet, J., 2003. The transverse force on a clean or contaminated bubble rising near a vertical wall at moderate Reynolds number. J. Fluid Mech. 495, 235-253.

Thuburn, J., 1996. Multidimensional flux-limited advection schemes. J. Comput. Phys. 123, 74-83.

Torres, D.J., Brackbill, J.U., 2000. The point-set method: front-tracking without connectivity. J. Comput. Phys. 165, 620-644.

Ubbink, O., Issa, R.I., 1999. A method for capturing sharp fluid interfaces on arbitrary meshes. J. Comput. Phys. 153, 26-50.

Unverdi, S., Tryggvason, G., 1992. A front-tracking method for viscous, incompressible multi-fluid flows. J. Comput. Phys. 100 , 25-37.

Youngs, D.L., 1982. Time-dependent multi-material flow with large fluid distortion. In: Morton, K.W., Baynes, M.J. (Eds.), Numerical Methods for Fluid Dynamics. Academic Press, New York, pp. 273-285.

Yuan, H., Prosperetti, A., 1994. On the in-line motion of two spherical bubbles in a viscous fluid. J. Fluid Mech. 278, 325-349.

Zalesak, S.T., 1979. Fully multidimensional Flux-Corrected Transport algorithms for fluids. J. Comput. Phys. 31, 335-362.

Zhou, H., Pozrikidis, C., 1993. The flow of ordered and random suspensions of two-dimensional drops in a channel. J. Fluid Mech. 255, $103-127$. 